\title{
Galerkin/Runge-Kutta Discretizations for Parabolic Equations with Time-Dependent Coefficients
}

\author{
By Stephen L. Keeling* \\ Dedicated to Professor Eugene Isaacson on the occasion of his 70 th birthday
}

\begin{abstract}
A new class of fully discrete Galerkin/Runge-Kutta methods is constructed and analyzed for linear parabolic initial-boundary value problems with time-dependent coefficients. Unlike any classical counterpart, this class offers arbitrarily high order of convergence while significantly avoiding what has been called order reduction. In support of this claim, error estimates are proved and computational results are presented. Additionally, since the time stepping equations involve coefficient matrices changing at each time step, a preconditioned iterative technique is used to solve the linear systems only approximately. Nevertheless, the resulting algorithm is shown to preserve the original convergence rate while using only the order of work required by the base scheme applied to a linear parabolic problem with time-independent coefficients. Furthermore, it is noted that special Runge-Kutta methods allow computations to be performed in parallel so that the final execution time can be reduced to that of a low-order method.
\end{abstract}

1. Introduction. In this paper, linear parabolic initial-boundary value problems with time-dependent coefficients are considered. Specifically, the goal is to construct and analyze fully discrete approximations to the unique solution $u(\mathbf{x}, t)$ of

$$
\begin{cases}\partial_{t} u=-L(t) u & \text { in } \Omega \times\left[0, t^{*}\right] \\ u=0 & \text { on } \partial \Omega \times\left[0, t^{*}\right] \\ x(\mathbf{x}, 0)=u^{0}(\mathbf{x}) & \text { in } \Omega\end{cases}
$$

where

$$
L(t) u \equiv-\sum_{i, j=1}^{N} \partial_{x_{i}}\left(\ell_{i j}(\mathbf{x}, t) \partial_{x_{j}} u\right)+\ell_{0}(\mathbf{x}, t) u .
$$

Here, $\Omega$ is a bounded domain in $\mathbf{R}^{N}$ with $\partial \Omega$ sufficiently smooth. Also, $\ell_{i j}(\mathbf{x}, t)$ and $\ell_{0}(\mathbf{x}, t)$ are assumed to be smooth. Further, on $\bar{\Omega} \times\left[0, t^{*}\right]$, the matrix $\left\{\ell_{i j}\right\}_{i, j=1}^{N}$ is symmetric and uniformly positive definite and $\ell_{0}$ is nonnegative. Also, the initial data $u^{0}$ is assumed to be both sufficiently smooth and compatible, and precise hypotheses on the required smoothness of the solution $u$ are made as needed.

Now, for $1 \leq p \leq \infty$ and integers $s \geq 0$, let $W^{s, p} \equiv W^{s, p}(\Omega)$ represent the well-known Sobolev spaces consisting of functions with (distributional) derivatives

Received September 14, 1987; revised April 15, 1988.

1980 Mathematics Subject Classification (1985 Revision). Primary 65M15.

Key words and phrases. Implicit Runge-Kutta methods, time-dependent coefficients, error estimates, order reduction.

*Supported by the National Aeronautics and Space Administration under NASA Contract No. NAS1-18107 while in residence at the Institute for Computer Applications in Science and Engineering (ICASE), NASA Langley Research Center, Hampton, VA 23665-5225. 
of order $\leq s$ in $L_{p} \equiv L_{p}(\Omega)$. Also, let $\|\cdot\|_{W^{s, p}}$ denote the usual norm. Then, in particular, take $H^{s} \equiv W^{s, 2}$ and denote its norm by $\|\cdot\|_{s}$. In addition, let $H_{0}^{1}$ be the subspace of $H^{1}$ consisting of functions vanishing on $\partial \Omega$ in the sense of trace. Further, let the inner product on $L_{2}$ be denoted by $(\cdot, \cdot)$, and the associated norm by $\|\cdot\|$. Next, given Hilbert spaces $H, H_{1}$, and $H_{2}, \mathscr{B}\left(H_{1}, H_{2}\right)$ represents the Hilbert space of bounded linear operators from $H_{1}$ into $H_{2}$, and $\mathscr{B}(H) \equiv \mathscr{B}(H, H)$. Also, for $t_{2}>t_{1}, \mathscr{C}^{l}\left(\left[t_{1}, t_{2}\right], H\right)$ denotes the Banach space of operators, continuously differentiable to order $l \geq 0$, from $\left[t_{1}, t_{2}\right]$ into $H$. See Adams [1] for more details.

Now for each $t \in\left[0, t^{*}\right]$, let $L(t)$ be extended to be $L_{2}$-selfadjoint with domain $H^{2} \cap H_{0}^{1}$. Also, assume that for $l \geq 0$ and $m \geq 0$ sufficiently large,

$$
L(t) \in \mathscr{C}^{l}\left(\left[0, t^{*}\right], \mathscr{B}\left(H^{m+2} \cap H_{0}^{1}, H^{m}\right)\right)
$$

so that

$$
\left\|L^{(l)}(t) v\right\|_{m} \leq c(l, m)\|v\|_{m+2} \quad \forall v \in H^{m+2} \cap H_{0}^{1},
$$

where $L^{(l)}(t) \equiv D_{t}^{l} L(t)$. Note that here and throughout this work, $c$ (sometimes with a subscript) is used to denote a general positive constant, not necessarily the same in any two places. Moreover, if in a given (in)equality there is a crucial element upon which $c$ is meant to depend, such dependence is indicated explicitly as in (1.2). Next, introducing the $L_{2}$-selfadjoint solution operator $T(t)$ for which $T(t) L(t)=I$ on $H^{2} \cap H_{0}^{1}$ and $L(t) T(t)=I$ on $L_{2}$, assume that for $l \geq 0$ and $m \geq 0$ sufficiently large, $T(t) \in \mathscr{C}^{l}\left(\left[0, t^{*}\right], \mathscr{B}\left(H^{m}, H^{m+2} \cap H_{0}^{1}\right)\right)$ so that

$$
\left\|T^{(l)}(t) v\right\|_{m+2} \leq c(l, m)\|v\|_{m} \quad \forall v \in H^{m}
$$

where $T^{(l)}(t) \equiv D_{t}^{l} T(t)$. Finally, assume that for sufficiently large $l \geq 0$ and $m \geq 0$, the solution $u$ and the data $u^{0}$ satisfy

$$
\sup _{0 \leq t \leq t^{*}}\left\|\partial_{t}^{l} u(t)\right\|_{m} \leq c(m, l)\left\|u^{0}\right\|_{m+2 l} .
$$

For details connected with (1.2)-(1.4), see Sammon [17].

A rough description of the results now follows. For this, let $h$ and $k$ denote spatial and temporal discretization parameters, respectively, and suppose that $U_{h}^{n}$ is a fully discrete approximation to $u(n k)$ obtained according to the base scheme (1.32) described below. In Section 3, the error committed by (1.32) is shown to satisfy

$$
\max _{0 \leq n \leq n^{*}}\left\|U_{h}^{n}-u^{n}\right\| \leq c\left(h^{r}+k^{\mu}+h k^{\mu-1 / 2}+h^{2} k^{\mu-1}\right)\left\|u^{0}\right\|_{\alpha}
$$

where $\alpha \equiv \max (r+1,2 \mu+2), \mu \equiv \min (\nu, q+1), q$ is the number of Runge-Kutta stages, and $r$ and $\nu$ represent, respectively, optimal exponents characteristic of the Galerkin method and the Runge-Kutta method upon which the fully discrete scheme is based. Note that under the mild condition that either $r \leq 2 \mu$ or $h^{2} \leq c k$, the above error is $\mathscr{O}\left(h^{r}+k^{\mu}\right)$. Further, it is explained below that the methods which are most easily implemented have the property that $\nu \leq q+1$, which makes the estimate optimal. It is also worth mentioning that inverse properties (associated with the use of a quasi-uniform triangulation of $\Omega$ ) are never explicitly assumed, and as explained after Proposition 3.3, the constructions of Section 2 are required for this. 
Next, Section 4 deals with (1.39), a variant of the base scheme which incorporates a preconditioned iterative method (PIM) for the time stepping equations (1.34). Specifically, these equations are solved only approximately at the $n$th time level with, say, $l_{n}$ outer iterations (4.3) and $j_{n}$ inner (PIM) iterations (4.8), and it is shown that the above convergence rate can be preserved while keeping $\sum_{n=0}^{n^{*}-1} l_{n} j_{n} / n^{*}$ bounded independently of $h$ and $k$. Hence, the order of work is asymptotically as that for a linear parabolic problem with time-independent coefficients. Additionally, in [15], semilinear and quasi-linear problems are considered, and the latter are treated with methods such as those reported here to obtain comparable results.

It should also be mentioned that the discovery of the methods described below was fortuitous. Note that there are extrapolation options other than (1.28) which are apparently more natural. For example, $D^{l}$ could be replaced by $T^{l}$ in $(1.28)$ since the latter is consistent with (1.33). This idea is considered together with (1.33) in a computational section. However, under rather general conditions, (1.5) is proved and demonstrated computationally only for (1.32) and (1.39). In fact, it has been reported by many authors ([7], [13], [8]) that unless the solution to the differential equation satisfies very restrictive conditions, a classical fully discrete scheme fashioned after (1.16) cannot be expected to offer optimal order of convergence. Furthermore, with regard to efficiency, (1.33) requires the formation of $q$ new stiffness matrices at every time step. On the other hand, (1.32) and (1.39) require only the formation of a single such matrix per time step and, at the expense of at most $100 q^{-1} \%$ more storage, the recall of $\mu-1$ of its counterparts formed at previous time steps.

In [7], Crouzeix analyzes (1.33), and with Butcher's conditions $C(p-1)$ and $B(\nu),[5]$, he establishes the $L_{2}$ estimate

$$
\max _{0 \leq n \leq n^{*}}\left\|U_{h}^{n}-u^{n}\right\|=\mathscr{O}\left(h^{r}+k^{\min (p, \nu)}\right) .
$$

Since $\mathscr{O}\left(h^{r}+k^{\nu}\right)$ has not generally been observed experimentally, this suboptimal phenomenon has been called order reduction. Note further that this $L_{2}$ estimate depends upon the assumption that the stages are computed exactly. On the other hand, in [13], Karakashian considers approximating the stages with a PIM and proves that the above estimate holds while the order of work is kept optimal. Also, he constructs collocation type implicit Runge-Kutta methods (IRKM's) for which $p=\nu=q+1$. Nevertheless, such methods have limited stability for $q \geq 3$. In fact, there is a general trade-off among IRKM's in the sense that the more stable methods suffer more from order reduction while those which do not suffer so, are not as stable. However, when (1.33) is modified as in (1.32), it is possible to achieve high order even for very stable methods. For example, in Section 4, an algebraically stable IRKM is used for a problem of the form (1.1), and optimal-order convergence is obtained with (1.39) but not with a counterpart based on (1.33).

Douglas, Dupont and Ewing [10] have analyzed Galerkin/Crank-Nicolson fully discrete approximations for a class of quasi-linear parabolic problems, proving an optimal $L_{2}$ estimate for a method which is second-order in time. Also, this rate was shown to be preserved by an algorithm in which the time stepping equations are solved only approximately with an optimal order of work. Then studying (1.1), 
Bramble and Sammon [3] have obtained similar results for some Galerkin/Obrechkoff fully discrete approximations, proving optimal $L_{2}$ estimates for methods up to fourth order in time. Finally, note that in [9], Dougalis and Karakashian analyze Galerkin/Runge-Kutta fully discrete approximations for the Korteweg-De Vries equation. In fact, they prove optimal $L_{2}$ estimates for some modified IRKM's which are up to fourth order. Hence, the spirit of their work is similar to that of the present study.

In the remainder of this section, there is a presentation of material relevant to the spatial and temporal discretizations considered here, which concludes with a precise definition of the schemes for which the above claims are made.

1.1. Spatial Discretizations. To make the following machinery more definite, consider the Ordinary Galerkin Method for the spatial approximation of the solution to $(1.1)$. Let $D(t)(\cdot, \cdot)$ be a bilinear form defined by

$$
D(t)(v, w) \equiv \sum_{i, j=1}^{N}\left(\ell_{i j}(t) \partial_{x_{i}} v, \partial_{x_{j}} w\right)+\left(\ell_{0}(t) v, w\right), \quad v, w \in H_{0}^{1}
$$

Next, let $S_{h}$ represent a finite-dimensional function space consisting of continuous, piecewise polynomials of degree $\leq r-1$, vanishing on $\partial \Omega$. Then, take $T_{h}(t): L_{2} \rightarrow$ $S_{h}$ to be an approximation to the solution operator $T(t)$, defined by

$$
D(t)\left(T_{h}(t) w, \chi\right) \equiv(w, \chi) \quad \forall w \in L_{2}, \forall \chi \in S_{h} .
$$

For more examples of Galerkin methods satisfying the assumptions enumerated below, see Bramble, Schatz, Thomée, and Wahlbin [4], and Sammon [17], [18].

Depending on the Galerkin method used, let $H_{E}$ be a linear space equipped with a norm $\|\cdot\|_{E}$ and satisfying the following properties. Suppose $H^{2} \cap H_{0}^{1} \subset H_{E}$ and that

$$
\|v\|_{1} \leq c\|v\|_{E} \quad \forall v \in H_{E}, \quad \text { and } \quad\|v\|_{E} \leq c\|v\|_{2} \quad \forall v \in H^{2} .
$$

For example, for the method described above, take $H_{E}=H_{0}^{1}$. Now let $\left\{S_{h}\right\}_{0<h<1}$ be a family of finite-dimensional subspaces of $H_{E}$ satisfying the following for some integer $r \geq 2$ :

$$
\inf _{\chi \in S_{h}}\left\{\|v-\chi\|+h\|v-\chi\|_{E}\right\} \leq c h^{s}\|v\|_{s} \quad \forall v \in H^{s} \cap H_{0}^{1}, 2 \leq s \leq r .
$$

Then suppose that for each $t \in\left[0, t^{*}\right]$, a family of operators $\left\{T_{h}(t)\right\}_{0<h<1}$ is given satisfying:

(i) $T_{h}(t): L_{2} \rightarrow S_{h}$ is selfadjoint, positive semidefinite on $L_{2}$, and positive definite on $S_{h}$,

(ii) For $0<h<1, T_{h}(t) \in \mathscr{C}^{l}\left(\left[0, t^{*}\right], \mathscr{B}\left(L_{2}, S_{h}\right)\right)$ for $l \geq 0$ sufficiently large,

(iii) For $2 \leq s \leq r, 0 \leq t \leq t^{*}$, and $l \geq 0$ as large as required in the sequel:

$$
\left\|\left[T^{(l)}(t)-T_{h}^{(l)}(t)\right] v\right\|+h\left\|\left[T^{(l)}(t)-T_{h}^{(l)}(t)\right] v\right\|_{E} \leq c h^{s}\|v\|_{s-2} \quad \forall v \in H^{s-2} .
$$

Hence, the restriction of $T_{h}(t)$ to $S_{h}$ is invertible and its inverse is henceforth denoted by $L_{h}(t)$. Since $L_{h}(t)$ is also positive definite and selfadjoint on $S_{h}$, both $L_{h}(t)$ and $T_{h}(t)$ have square roots, but it is also assumed that

$$
\begin{aligned}
c\left\|T_{h}^{1 / 2}(t) \chi\right\| & \leq c\left\|T_{h}^{1 / 2}(t) \chi\right\|_{E} \leq\|\chi\| \leq\|\chi\|_{E} \\
& \leq c\left\|L_{h}^{1 / 2}(t) \chi\right\| \leq c\left\|L_{h}^{1 / 2}(t) \chi\right\|_{E} \quad \forall \chi \in S_{h} .
\end{aligned}
$$


Also, $L_{h}(t) \in \mathscr{C}^{l}\left(\left[0, t^{*}\right], \mathscr{B}\left(S_{h}\right)\right)$ for $l \geq 0$ sufficiently large and in fact, Bales [2] has proved that for $0 \leq s, t \leq t^{*}$, and $l \geq 0$ :

$$
\left\|L_{h}^{1 / 2}(t) T_{h}^{(l)}(s) L_{h}^{1 / 2}(t) \chi\right\|+\left\|T_{h}^{1 / 2}(t) L_{h}^{(l)}(s) T_{h}^{1 / 2}(t) \chi\right\| \leq c(l)\|\chi\| \quad \forall \chi \in S_{h} .
$$

Then using the selfadjointness of these operators, the following are straightforward consequences of (1.8). For $0 \leq s, t \leq t^{*}$,

$$
\begin{gathered}
\left\|T_{h}^{1 / 2}(s) L_{h}^{1 / 2}(t) \chi\right\|+\left\|L_{h}^{1 / 2}(s) T_{h}^{1 / 2}(t) \chi\right\| \leq c\|\chi\| \quad \forall \chi \in S_{h}, \\
\left|\left(L_{h}^{(l)}(s) \chi, \chi\right)\right| \leq c(l)\left(L_{h}(t) \chi, \chi\right) \quad \forall \chi \in S_{h} .
\end{gathered}
$$

In addition to (1.10), assume that for $0 \leq t \leq t^{*}$ and $l \geq 0$,

$$
\left|\left(L_{h}^{(l)}(t) \chi, \phi\right)\right| \leq c(l)\|\chi\|_{E}\|\phi\|_{E} \quad \forall \chi, \phi \in S_{h} .
$$

Next, defining the elliptic projection operator as $P_{E}(t) \equiv T_{h}(t) L(t)$, it follows from (1.6) and (1.2) that for $0 \leq t \leq t^{*}$,

(1.11) $\left\|\left[I-P_{E}(t)\right] v\right\|+h\left\|\left[I-P_{E}(t)\right] v\right\|_{E} \leq c h^{s}\|v\|_{s} \quad \forall v \in H^{s} \cap H_{0}^{1}, 2 \leq s \leq r$.

In fact, with $\omega(t) \equiv P_{E}(t) u(t)$ and $\eta(t) \equiv u(t)-\omega(t),(1.2),(1.4)$, and (1.6) can be used [3] to show that

$$
\sup _{0 \leq t \leq t^{*}}\left\{\left\|\eta^{(l)}(t)\right\|+h\left\|\eta^{(l)}(t)\right\|_{E}\right\} \leq c(l) h^{s}\left\|u^{0}\right\|_{s+2 l}, \quad 2 \leq s \leq r .
$$

Finally, it can be shown that $P_{0} \equiv L_{h}(t) T_{h}(t)$ is for every $t \in\left[0, t^{*}\right]$ the orthogonal projection of $L_{2}$ onto $S_{h}$ and that $T_{h}(t)=T_{h}(t) P_{0}$. Then, since $I-P_{0}$ is majorized by $I-P_{E}$ in $L_{2}$, it follows from (1.11) that

$$
\left\|\left(I-P_{0}\right) v\right\| \leq c h^{s}\|v\|_{s} \quad \forall v \in H^{s} \cap H_{0}^{1}, 2 \leq s \leq r .
$$

Now, (1.1) has the following semidiscrete formulation. Find $u_{h}:\left[0, t^{*}\right] \rightarrow S_{h}$ satisfying

$$
\left\{\begin{aligned}
\partial_{t} u_{h} & =-L_{h}(t) u_{h}, \\
u_{h}(0) & =u_{h}^{0},
\end{aligned}\right.
$$

where $u_{h}^{0} \in S_{h}$ is a suitable approximation to $u^{0}$. In [18], Sammon analyzes approximations of the form (1.14), and with assumptions comparable to those described above, he proves an optimal $L_{2}$ estimate

$$
\sup _{0 \leq t \leq t^{*}}\left\|u(t)-u_{h}(t)\right\| \leq c h^{r}\left\|u^{0}\right\|_{r} .
$$

In the present paper, semidiscrete approximations are not analyzed. Instead, (1.14) serves only as a source of inspiration for fully discrete approximations, and $u_{h}$ does not appear in forthcoming proofs.

1.2. Temporal Discretizations. For the temporal approximation of the solution to (1.14), Implicit Runge-Kutta Methods (IRKM's) are now introduced. Given an integer $q \geq 1$, a $q$-stage IRKM is characterized by a set of constants $\left\{a_{i j}\right\}_{i, j=1}^{q}$, $\left\{b_{j}\right\}_{j=1}^{q}$, and $\left\{\tau_{i}\right\}_{i=1}^{q}$, and it is convenient to make the following definitions:

$$
\begin{gathered}
A \equiv\left\{a_{i j}\right\}_{1 \leq i, j \leq q}, \quad b \equiv\left\langle b_{1}, b_{2}, \ldots, b_{q}\right\rangle^{T}, \quad B \equiv \operatorname{diag}_{1 \leq i \leq q}\left\{b_{i}\right\} \\
M \equiv B A+A^{T} B-b b^{T}, \quad T \equiv \operatorname{diag}_{1 \leq i \leq q}\left\{\tau_{i}\right\}, \quad e \equiv\langle 1,1, \ldots, 1\rangle^{T} .
\end{gathered}
$$


For the IRKM formulation used in this work, choose arbitrarily $t_{0} \in \mathbf{R}, \mathbf{y}_{0} \in \mathbf{R}^{m}$, $\mathbf{F}: \mathbf{R}^{m+1} \rightarrow \mathbf{R}^{m}$ sufficiently smooth, and $k>0$ sufficiently small, so that for $t_{0} \leq t \leq t_{0}+k$, smooth functions $\mathbf{y}, \hat{\mathbf{y}}: \mathbf{R} \rightarrow \mathbf{R}^{m}$ are well defined by

$$
\begin{gathered}
\left\{\begin{array}{c}
D_{t} \mathbf{y}(t)=\mathbf{F}(t, \mathbf{y}(t)), \\
\mathbf{y}\left(t_{0}\right)=\mathbf{y}_{0},
\end{array}\right. \\
\left\{\begin{array}{l}
\mathbf{y}^{i}(t)=\mathbf{y}_{0}+\left(t-t_{0}\right) \sum_{j=1}^{q} a_{i j} \mathbf{F}\left(t_{0}+\tau_{j}\left(t-t_{0}\right), \mathbf{y}^{j}(t)\right), \quad 1 \leq i \leq q, \\
\hat{\mathbf{y}}(t)=\mathbf{y}_{0}+\left(t-t_{0}\right) \sum_{i=1}^{q} b_{i} \mathbf{F}\left(t_{0}+\tau_{i}\left(t-t_{0}\right), \mathbf{y}^{i}(t)\right) .
\end{array}\right.
\end{gathered}
$$

The method is described as explicit if $a_{i j}=0, i \leq j$, and implicit if for any $i$, $a_{i i} \neq 0$. Also, it is said to have order $\nu$ if for every $\mathbf{y}$ and $\hat{\mathbf{y}}$ defined as above, $D_{t}^{l} \mathbf{y}\left(t_{0}\right)=D_{t}^{l} \hat{\mathbf{y}}\left(t_{0}\right), 0 \leq l \leq \nu$. Butcher [5] has developed simple conditions for the above parameters which guarantee a given order; however, only the following is explicitly required in this work:

$$
l ! b^{T} A^{l-1} e=1, \quad 1 \leq l \leq \nu .
$$

To see the roots of condition (1.17), let (1.15) have $m=1, t_{0}=0, y_{0}=1$, and $F(y)=-y$, so that $y(t)=e^{-t}$. Then, from (1.16), $\hat{y}(t)=r(t)$, where $r(z)$ is a rational approximation to the exponential $e^{-z}$ given by

$$
r(z) \equiv 1-z b^{T}(I+z A)^{-1} e .
$$

Expanding this expression shows that $r(z)$ is a $\nu$ th-order approximation to the exponential if and only if (1.17) holds. Next, with regard to stability, an IRKM is said to be $A_{0}$-stable if

$$
|r(z)| \leq 1 \quad \forall z \geq 0
$$

and strongly $A_{0}$-stable if

$$
\sup _{z \geq z_{0}}|r(z)|<1 \quad \forall z_{0}>0
$$

Also, a method is called algebraically stable if $M$ and $B$ are positive semidefinite. However, if an algebraically stable method is irreducible (not equivalent to a fewerstage method), then

$B$ is positive definite, and $M$ is positive semidefinite.

One other notion of stability which is useful here is that of dissipativity:

$$
-1<-1+\delta \leq r(z) \leq 1 \quad \forall z \geq 0 .
$$

$A_{0}$-stability is required of all IRKM's considered in this work. However, in order for the approximations to decay with respect to the time step, strong $A_{0}$-stability must hold. In fact, to guarantee decay, both (1.20) and (1.21) are assumed. Then in Section 4, the iterative scheme (1.39) described below requires at least (1.22) in addition to

$$
r(z) \leq 1-c \frac{z}{(1+z)^{3}} \quad \forall z \geq 0
$$


This growth condition is extremely mild, and this author is unaware of any popular IRKM which fails to satisfy it. Also, requiring (1.22) and (1.23) improves on a related result of Karakashian [13] in which (1.20) is used. Next, note that the spectrum of $A, \sigma(A)$, is related to the poles of $r(z)$, and in addition to the above, it is assumed throughout this paper that

$$
\sigma(A) \subset\{x \in \mathbf{R}: x>0\} .
$$

Returning to the temporal discretization of (1.14), let a $q$-stage IRKM of order $\nu \geq 1$ be given. Assume also that there exists a $q \times q$ matrix $D$ satisfying:

$$
D\left[e ; A e ; \ldots ; A^{q-1} e\right]=\left[A e ; 2 A^{2} e ; \ldots ; q A^{q} e\right] .
$$

Again, this author is unaware of any well-known IRKM for which such a $D$ fails to exist. In fact, the so-called collocation type methods are those for which $D=T$. Now with $\mu \equiv \min (\nu, q+1)$, it follows from (1.25) and (1.17) that

$$
\begin{gathered}
l A D^{l-1} e=D^{l} e, \quad 1 \leq l \leq \mu-1, \\
l b^{T} D^{l-1} e=1, \quad 1 \leq l \leq \mu .
\end{gathered}
$$

Next, for $0 \leq n \leq n^{*}-1, n^{*} k \equiv t^{*}$, let the real values $\left\{\delta_{m}^{n}\right\}_{m=0}^{\mu-1}$ be chosen distinctly so that the $q \times q$ matrices $\left\{\Gamma_{m}^{n}\right\}_{m=0}^{\mu-1}$ are well defined by

$$
\sum_{m=0}^{\mu-1} \Gamma_{m}^{n}\left(\delta_{m}^{n}\right)^{l}=D^{l}, \quad 0 \leq l \leq \mu-1,
$$

as the computation of their components involves the inversion of the $\mu \times \mu$ Vandermonde matrix $\left\{\left(\delta_{m}^{n}\right)^{l}\right\}_{m, l=0}^{\mu-1}$. In addition, assume that these parameters are bounded independently of $n$ :

$$
\max _{0 \leq m \leq \mu-1}\left\{\left|\delta_{m}^{n}\right|+\max _{1 \leq i, j \leq q}\left|\left(\Gamma_{m}^{n}\right)_{i j}\right|\right\} \leq c .
$$

Actually, it is clear below that the natural and computationally advantageous choice for $(1.28)$ is:

$$
\delta_{m}^{n}= \begin{cases}m-n, & 0 \leq n \leq \mu-2, \\ -m, & \mu-1 \leq n \leq n^{*}-1 .\end{cases}
$$

In any case, define $t^{n} \equiv n k$ and $\tau_{m}^{n} \equiv t^{n}+\delta_{m}^{n} k$, and for $0 \leq n \leq n^{*}-1,0 \leq t \leq t^{*}$, and $0 \leq s \leq k$, let the following be defined on $\mathbf{S}_{h} \equiv\left[S_{h}\right]^{q}$ :

$$
\begin{gathered}
\mathscr{L}_{h}(t) \equiv \operatorname{diag}_{q \times q}\left\{L_{h}(t)\right\}, \quad \mathscr{L}_{h}^{n} \equiv \mathscr{L}_{h}\left(t^{n}\right), \\
\overline{\mathscr{L}}_{h}^{n}(s) \equiv \sum_{m=0}^{\mu-1} \Gamma_{m}^{n} \mathscr{L}_{h}\left(t^{n}+\delta_{m}^{n} s\right), \quad \overline{\mathscr{L}}_{h}^{n} \equiv \overline{\mathscr{L}}_{h}^{n}(k) .
\end{gathered}
$$

Now with

$$
U_{h}^{0}=\left[I+k L_{h}^{0}\right]^{-1} P_{0}\left[I+k L^{0}\right] u^{0},
$$

suppose that for $0 \leq n \leq n^{*}-1$ the approximation $U_{h}^{n} \in S_{h}$ is given, where $U_{h}^{n} \approx u^{n}$ and $u^{n} \equiv u\left(\mathbf{x}, t^{n}\right)$. Then, let $U_{h}^{n+1} \approx u^{n+1}$ be given by what is henceforth called the base scheme:

$$
\left\{\begin{aligned}
\bar{U}_{h}^{n} & =e U_{h}^{n}-k A \overline{\mathscr{L}}_{h}^{n} \bar{U}_{h}^{n}, \\
U_{h}^{n+1} & =\left(I-b^{T} A^{-1} e\right) U_{h}^{n}+b^{T} A^{-1} \bar{U}_{h}^{n},
\end{aligned}\right.
$$


where $\bar{U}_{h}^{n} \in \mathbf{S}_{h}$ is well defined provided $\left[I+k A \overline{\mathscr{L}}_{h}^{n}\right]$ is invertible. Here, $A \overline{\mathscr{L}}_{h}^{n}$, for example, is understood in the sense of composition of operators defined on $\mathbf{S}_{h}$. Note that if the temporal discretization of (1.14) were accomplished as prescribed by (1.15) and (1.16), the following would result:

$$
\left\{\begin{aligned}
\bar{U}_{h}^{n} & =e U_{h}^{n}-k A \tilde{\mathscr{L}}_{h}^{n} \bar{U}_{h}^{n}, \\
U_{h}^{n+1} & =\left(I-b^{T} A^{-1} e\right) U_{h}^{n}+b^{T} A^{-1} \bar{U}_{h}^{n}, \\
& \text { where } \tilde{\mathscr{L}}_{h}^{n} \equiv \operatorname{diag}_{1 \leq i \leq q}\left\{L_{h}\left(t^{n}+k \tau_{i}\right)\right\} .
\end{aligned}\right.
$$

However, as discussed in the beginning of the Introduction, (1.32) is designed to improve upon (1.33) with the indicated modification.

Now, with regard to iterative approximations, note that an efficient method is needed for solving the time stepping equations

$$
\left[I+k A \overline{\mathscr{L}}_{h}^{n}\right] \bar{U}_{h}^{n}=e U_{h}^{n} \text {. }
$$

According to (1.24), $A$ can be transformed as follows:

$$
\begin{array}{ll}
S A S^{-1}=\Lambda \equiv \underset{1 \leq i \leq q}{\operatorname{diag}}\left\{\lambda_{i}\right\}+\underset{2 \leq i \leq q}{\operatorname{subdiag}}\left\{\theta_{i}\right\}, & \lambda_{i}>0,1 \leq i \leq q, \\
& \theta_{i}=0 \text { or } 1,2 \leq i \leq q .
\end{array}
$$

Then $\bar{V}_{l_{n}}^{n} \approx \bar{U}_{h}^{n}$ can be obtained by the (outer) iterations

$$
\left[I+k \Lambda \mathscr{L}_{h}^{n}\right]\left(S \bar{V}_{l}^{n}\right)=\left\{S e U_{h}^{n}+k S A\left(\mathscr{L}_{h}^{n}-\overline{\mathscr{L}}_{h}^{n}\right) \bar{V}_{l-1}^{n}\right\} \equiv R_{l}^{n}, \quad 1 \leq l \leq l_{n}
$$

where

$$
\begin{aligned}
\tilde{V}_{0}^{n} \equiv \sum_{m=n-1-\mu_{n}}^{n-1}(-1)^{n-m-1}\left(\begin{array}{l}
\mu_{n}+1 \\
n-m
\end{array}\right) \tilde{U}_{h}^{m}, \quad 1 \leq n \leq n^{*}-1, \tilde{V}_{0}^{0} \equiv e U_{h}^{0}, \\
l_{n} \equiv \begin{cases}\mu, & n=0, \\
\mu+1-n, & 1 \leq n \leq \mu, \\
1, & \mu+1 \leq n \leq n^{*}-1,\end{cases} \\
\mu_{n} \equiv \begin{cases}0, & n=0, \\
n-1, & 1 \leq n \leq \mu, \\
\mu, & \mu+1 \leq n \leq n^{*}-1,\end{cases}
\end{aligned}
$$

and (1.35) is started with $\bar{V}_{0}^{n} \equiv \tilde{V}_{0}^{n}$ provided $\left\{\tilde{U}_{h}^{m}\right\}_{m=n-1-\mu_{n}}^{n-1}$ are computed as indicated below. Now consider the simple but important observation that if

$$
\lambda_{i} \neq \lambda_{j}, \quad i \neq j \quad \text { and } \quad \theta_{i}=0, \quad 2 \leq i \leq q
$$

then the block system above decouples into the following equations, which can be solved in parallel:

$$
\left[I+k \lambda_{i} L_{h}^{n}\right]\left(S \bar{V}_{l}^{n}\right)_{i}=\left(R_{l}^{n}\right)_{i}, \quad 1 \leq i \leq q .
$$

Then, to avoid having to factor new coefficient matrices at every time step, a preconditioned iterative method is used to approximate $\bar{V}_{l}^{n}$ with (inner) iterates, say $\left\{\tilde{V}_{l, j}^{n}\right\}_{0 \leq j \leq j_{n}}$. Further, it is shown that there exist integers $\left\{j_{n}\right\}_{n=0}^{n^{*}-1}$ such 
that $\sum_{n=0}^{n^{*}-1} l_{n} j_{n} / n^{*} \leq c$ while the convergence order (1.5) is preserved for what is henceforth called the iterative scheme:

$$
\left\{\begin{aligned}
\tilde{U}_{h}^{n} & =\tilde{V}_{l_{n}, j_{n}}^{n} \\
U_{h}^{n+1} & =\left(I-b^{T} A^{-1} e\right) U_{h}^{n}+b^{T} A^{-1} \tilde{U}_{h}^{n} .
\end{aligned}\right.
$$

Finally, let the initial approximation for this scheme be given by (1.31) also.

2. The Product Space Operators. In this section, the machinery elaborated between (1.2) and (1.13) is generalized to analogous operators defined on products of spaces on which their precursors are defined. Also, certain technical lemmas are established for later use. Now, in addition to $\mathbf{S}_{h}$, define the product spaces $\mathbf{L}_{2} \equiv\left[L_{2}\right]^{q}, \mathbf{H}_{0}^{1} \equiv\left[H_{0}^{1}\right]^{q}, \mathbf{H}_{E} \equiv\left[H_{E}\right]^{q}$, and $\mathbf{H}^{m}=\left[H^{m}\right]^{q}$. Also, on these spaces define

$$
\begin{gathered}
\|\boldsymbol{\Phi}\|_{E} \equiv\left\{\sum_{i=1}^{q}\left\|\phi_{i}\right\|_{E}^{2}\right\}^{1 / 2}, \quad\|\boldsymbol{\Phi}\|_{m} \equiv\left\{\sum_{i=1}^{q}\left\|\phi_{i}\right\|_{m}^{2}\right\}^{1 / 2}, \\
(\boldsymbol{\Phi}, \boldsymbol{\Psi}) \equiv \sum_{i=1}^{q}\left(\phi_{i}, \psi_{i}\right), \quad\|\boldsymbol{\Phi}\|=(\boldsymbol{\Phi}, \boldsymbol{\Phi})^{1 / 2} .
\end{gathered}
$$

Then, for $0 \leq n \leq n^{*}-1,0 \leq t \leq t^{*}$ and $0 \leq s \leq k$, let the following be defined on $\mathbf{H}^{2} \cap \mathbf{H}_{0}^{1}$ :

$$
\begin{gathered}
\mathscr{L}(t)=\operatorname{diag}_{q \times q}\{L(t)\}, \quad \mathscr{L}^{n} \equiv \mathscr{L}\left(t^{n}\right), \\
\overline{\mathscr{L}}^{n}(s) \equiv \sum_{m=0}^{\mu-1} \Gamma_{m}^{n} \mathscr{L}\left(t^{n}+\delta_{m}^{n} s\right), \quad \overline{\mathscr{L}}^{n} \equiv \overline{\mathscr{L}}^{n}(k) .
\end{gathered}
$$

The first step is to construct, for $0 \leq n \leq n^{*}-1$ and $0 \leq s \leq k$, operators $\overline{\mathscr{T}}^{n}(s)$ $\left(\overline{\mathscr{T}}^{n} \equiv \overline{\mathscr{T}}^{n}(k)\right)$ satisfying

$$
\begin{cases}\overline{\mathscr{L}}^{n}(s) \overline{\mathscr{T}}^{n}(s)=I & \text { on } \mathbf{L}_{2}, \\ \overline{\mathscr{T}}^{n}(s) \overline{\mathscr{L}}^{n}(s)=I & \text { on } \mathbf{H}^{2} \cap \mathbf{H}_{0}^{1} .\end{cases}
$$

Note that with $\mathscr{T}(t) \equiv \operatorname{diag}_{q \times q}\{T(t)\}$ and $\mathscr{T}^{n} \equiv \mathscr{T}\left(t^{n}\right)$ defined on $\mathbf{L}_{2}$ for $0 \leq t \leq$ $t^{*}$ and $0 \leq n \leq n^{*}-1, \overline{\mathscr{T}}^{n}(s)$ cannot be taken as a combination of such operators.

LEMMA 2.1. For $0 \leq n \leq n^{*}-1, \overline{\mathscr{L}}^{n}(s) \in \mathscr{C}^{l}\left([0, k], \mathscr{B}\left(\mathbf{H}^{m+2} \cap \mathbf{H}_{0}^{1}, \mathbf{H}^{m}\right)\right)$, where $l, m \geq 0$ are as in (1.2). Also the following hold for all $\mathbf{v} \in \mathbf{H}^{m+2} \cap \mathbf{H}_{0}^{1}$, $0 \leq s \leq k$, and $0 \leq n \leq n^{*}-1$ :

$$
\begin{gathered}
\left\|\partial_{s}^{l} \overline{\mathscr{L}}^{n}(s) \mathbf{v}\right\|_{m} \leq c(l, m)\|\mathbf{v}\|_{m+2} \\
\left\|\left[\overline{\mathscr{L}}^{n}(s)-\mathscr{L}^{n}\right] \mathbf{v}\right\|_{m} \leq c(m) k\|\mathbf{v}\|_{m+2} .
\end{gathered}
$$

Proof. The crucial observation is that by (1.28) with $l=0$ :

$$
\overline{\mathscr{L}}^{n}(s)-\mathscr{L}^{n}=\sum_{i=0}^{\mu-1} \Gamma_{i}^{n}\left[\mathscr{L}\left(t^{n}+\delta_{i}^{n} s\right)-\mathscr{L}^{n}\right]=\sum_{i=0}^{\mu-1} \Gamma_{i}^{n} \int_{t^{n}}^{t^{n}+\delta_{i}^{n} s} \mathscr{L}^{(1)}(t) d t,
$$

and (2.3) follows with (1.29) and (1.2). Also, (2.2) follows using (1.29) and (1.2). 
THEOREM 2.1. Let $m, l \geq 0$ be as in (1.3). Then for $k$ small enough, and $0 \leq n \leq n^{*}-1$, there exist operators $\overline{\mathscr{T}}^{n}(s) \in \mathscr{C}^{l}\left([0, k], \mathscr{B}\left(\mathbf{H}^{m}, \mathbf{H}^{m+2} \cap \mathbf{H}_{0}^{1}\right)\right)$ satisfying (2.1) and

$$
\left\|\partial_{s}^{l} \overline{\mathscr{T}}^{n}(s) \mathbf{v}\right\|_{m+2} \leq c(l, m)\|\mathbf{v}\|_{m} \quad \forall \mathbf{v} \in \mathbf{H}^{m} .
$$

Proof. The details appear in [14]. However, the key idea is to use (2.3) to show that with $\mathbf{v} \in \mathbf{H}^{m}, \mathscr{F} \mathbf{u} \equiv \mathscr{T}^{n}\left\{\mathbf{v}+\left[\mathscr{L}^{n}-\overline{\mathscr{L}}^{n}(s)\right] \mathbf{u}\right\}$ is a global contraction on $\mathbf{H}^{m+2} \cap \mathbf{H}_{0}^{1}$, provided $k$ is small enough. Then the unique fixed point is taken to be $\overline{\mathscr{T}}^{n}(s) \mathbf{v}$, and (2.4) is established inductively.

The next step is to construct for $0 \leq n \leq n^{*}-1$ and $0 \leq s \leq k$ operators $\overline{\mathscr{T}}_{h}^{n}(s)$ $\left(\overline{\mathscr{T}}_{h}^{n} \equiv \overline{\mathscr{T}}_{h}^{n}(k)\right)$ satisfying

$$
\begin{cases}\overline{\mathscr{L}}_{h}^{n}(s) \overline{\mathscr{T}}_{h}^{n}(s)=\mathscr{P}_{0} & \text { on } \mathbf{L}_{2} \\ \overline{\mathscr{T}}_{h}^{n}(s) \overline{\mathscr{L}}_{h}^{n}(s)=I & \text { on } \mathbf{S}_{h}\end{cases}
$$

where $\mathscr{P}_{0} \equiv \operatorname{diag}_{q \times q}\left\{P_{0}\right\}$. Note that with $\mathscr{T}_{h}(t) \equiv \operatorname{diag}_{q \times q}\left\{T_{h}(t)\right\}$ and $\mathscr{T}_{h}^{n} \equiv$ $\mathscr{T}_{h}\left(t^{n}\right)$ defined on $\mathrm{L}_{2}$ for $0 \leq t \leq t^{*}$ and $0 \leq n \leq n^{*}-1, \overline{\mathscr{T}}_{h}^{n}(s)$ cannot be taken as a combination of such operators.

Now let $\left\{D_{h}(t)(\cdot, \cdot)\right\}_{0 \leq t \leq t^{*}}$ be a family of bilinear forms defined on $H_{E} \times H_{E}$ so that

$$
D_{h}(t)(\chi, \phi)=\left(L_{h}(t) \chi, \phi\right) \quad \forall \chi, \phi \in S_{h} .
$$

More specifically, with $D_{h}^{(l)}(t)(\cdot, \cdot) \equiv D_{t}^{l} D_{h}(\cdot, \cdot)$, assume that for $0 \leq t \leq t^{*}, l \geq 0$, and $2 \leq m \leq r$,

$$
\begin{gathered}
\left|D_{h}^{(l)}(t)(v, w)-\left(L^{(l)}(t) v, w\right)\right| \leq c(l) h^{m-1}\|v\|_{m}\|w-u\|_{E} \\
\forall v \in H^{m} \cap H_{0}^{1}, \forall w \in H^{2} \cap H_{0}^{1}+S_{h}, \forall u \in H^{2} \cap H_{0}^{1}, \\
\left|D_{h}^{(l)}(t)(w, v)\right| \leq c(l)\|w\|_{E}\|v\|_{E} \quad \forall w, v \in H_{E}, \\
c\|\chi\|_{E}^{2} \leq D_{h}(t)(\chi, \chi) \quad \forall \chi \in S_{h} .
\end{gathered}
$$

For example, these assumptions are readily verified for the Ordinary Galerkin Method mentioned in the Introduction. For additional examples, see Sammon [17], [18]. Next, for $0 \leq t \leq t^{*}, 0 \leq n \leq n^{*}-1$ and $0 \leq s \leq k$, let the following be defined on $\mathbf{H}_{E} \times \mathbf{H}_{E}$ :

$$
\mathscr{D}_{h}(t)(\mathbf{w}, \mathbf{v}) \equiv \sum_{i=1}^{q} D_{h}(t)\left(w_{i}, v_{i}\right), \quad \overline{\mathscr{D}}_{h}^{n}(s)(\mathbf{w}, \mathbf{v}) \equiv \sum_{m=0}^{\mu-1} \mathscr{D}_{h}\left(t^{n}+\delta_{m}^{s} s\right)\left(\Gamma_{m}^{n} \mathbf{w}, \mathbf{v}\right)
$$

Discrete counterparts to Lemma 2.1 and Theorem 2.1 appear next.

LEMMA 2.2. For $0 \leq n \leq n^{*}-1, \overline{\mathscr{L}}_{h}^{n}(s) \in \mathscr{C}^{l}\left([0, k], \mathscr{B}\left(\mathbf{S}_{h}\right)\right)$ where $l \geq 0$ is as in (1.8). Also, the following hold for all $\mathbf{f} \in \mathrm{L}_{2}, 0 \leq t, t_{1}, t_{2} \leq t^{*}, 0 \leq s \leq k$, $0 \leq n \leq n^{*}-1$ :

$$
\begin{gathered}
\left\|\mathscr{T}_{h}^{1 / 2}(t) \partial_{s}^{l} \overline{\mathscr{L}}_{h}^{n}(s) \mathscr{T}_{h}^{1 / 2}(t) \mathbf{f}\right\| \leq c(l)\|\mathbf{f}\|, \\
\left\|\mathscr{T}_{h}^{1 / 2}(t)\left[\mathscr{L}_{h}\left(t_{2}\right)-\mathscr{L}_{h}\left(t_{1}\right)\right] \mathscr{T}_{h}^{1 / 2}(t) \mathbf{f}\right\| \leq c\left|t_{2}-t_{1}\right|\|\mathbf{f}\|, \\
\left\|\mathscr{T}_{h}^{1 / 2}(t)\left[\overline{\mathscr{L}}_{h}^{n}(s)-\mathscr{L}_{h}^{n}\right] \mathscr{T}_{h}^{1 / 2}(t) \mathbf{f}\right\| \leq c k\|\mathbf{f}\| .
\end{gathered}
$$

Proof. The manipulations required are similar to those needed for Lemma 2.1, except that (1.8) is used instead of (1.2). 
THEOREM 2.2. Let $l \geq 0$ be as in (1.8). Then for $k$ small enough, and $0 \leq$ $n \leq n^{*}-1$, there exist operators $\overline{\mathscr{T}}_{h}^{n}(s) \in \mathscr{C}^{l}\left([0, k], \mathscr{B}\left(\mathbf{L}_{2}, \mathbf{S}_{h}\right)\right)$ defined by

$$
\overline{\mathscr{D}}_{h}^{n}(s)\left(\overline{\mathscr{T}}_{h}^{n}(s) \mathbf{f}, \mathbf{X}\right)=(\mathbf{f}, \mathbf{X}) \quad \forall \mathbf{f} \in \mathbf{L}_{2}, \forall \mathbf{X} \in \mathbf{S}_{h}, 0 \leq s \leq k
$$

and satisfying (2.5) and, in addition, for all $\mathbf{f} \in \mathbf{L}_{2}$, for all $\mathbf{X} \in \mathbf{S}_{h}, \theta_{1}, \theta_{2}=0, \frac{1}{2}$, $0 \leq t \leq t^{*}, 0 \leq s \leq k, 0 \leq n \leq n^{*}-1$,

$$
\begin{gathered}
\left\|\partial_{s}^{l} \overline{\mathscr{T}}_{h}^{n}(s) \mathbf{f}\right\|_{E} \leq c(l)\|\mathbf{f}\|, \\
\left\|\mathscr{L}_{h}^{\theta_{1}}(t) \partial_{s}^{l} \overline{\mathscr{T}}_{h}^{n}(s) \mathscr{L}_{h}^{\theta_{2}}(t) \mathbf{X}\right\| \leq c(l)\|\mathbf{X}\| .
\end{gathered}
$$

Proof. The details appear in [14]. However, that $\overline{\mathscr{T}}_{h}^{n}(s)$ is well defined by (2.9) follows once $\overline{\mathscr{D}}_{h}^{n}(s)$ is shown to satisfy the hypotheses of the Lax-Milgram Lemma [6]. Then, (2.10) and (2.11) are established inductively using (1.7), (1.9), (1.28), (1.29), (2.6), and (2.8).

Finally, a generalization of (1.6) is required for the proof of Lemma 3.3.

THEOREM 2.3. For $k>0$ small enough, the following holds for $0 \leq n \leq n^{*}-1$, $0 \leq s \leq k, l \geq 0$, and $2 \leq m \leq r$ :

$\left\|\partial_{s}^{l}\left[\overline{\mathscr{T}}^{n}(s)-\overline{\mathscr{T}}_{h}^{n}(s)\right] \mathbf{v}\right\|+h\left\|\partial_{s}^{l}\left[\overline{\mathscr{T}}^{n}(s)-\overline{\mathscr{T}}_{h}^{n}(s)\right] \mathbf{v}\right\|_{E} \leq c(l) h^{m}\|\mathbf{v}\|_{m-2} \quad \forall \mathbf{v} \in \mathbf{H}^{m-2}$.

Proof. See [14].

3. The Base Scheme. In this section, the base scheme (1.32) is analyzed for the approximation of the solution to (1.1), and (1.5) is established. That the stages are well defined depends on the next lemma.

LEMMA 3.1. Provided (1.24) is satisfied, $\left[I+k A \mathscr{L}_{h}^{n}\right]$ is invertible, and for $k$ small enough, $\left[I+k A \overline{\mathscr{L}}_{h}^{n}\right]$ is as well. Also the following hold for all $\mathbf{X} \in \mathbf{S}_{h}$, $0 \leq \theta \leq 1, \theta_{1}, \theta_{2}=0, \frac{1}{2}, \theta_{1}=-\theta_{2}= \pm \frac{1}{2}, 0 \leq n \leq n^{*}-1$ :

$$
\begin{gathered}
\left\|\left(k \mathscr{L}_{h}^{n}\right)^{\theta}\left[I+k A \mathscr{L}_{h}^{n}\right]^{-1} \mathbf{X}\right\| \leq c\|\mathbf{X}\|, \\
\left\|\left(k \mathscr{L}_{h}^{n}\right)^{\theta_{1}}\left[I+k A \overline{\mathscr{L}}_{h}^{n}\right]^{-1}\left(k \mathscr{L}_{h}^{n}\right)^{\theta_{2}} \mathbf{X}\right\| \leq c\|\mathbf{X}\| .
\end{gathered}
$$

Proof. The invertibility of $\left[I+k A \mathscr{L}_{h}^{n}\right]$ and the estimate (3.1) involve a spectral argument after $\boldsymbol{A}$ is transformed to Jordan form, and the details are provided by Karakashian [13]. Now set

$E_{1} \equiv\left[I+k A \mathscr{L}_{h}^{n}\right]^{-1} k A\left(\mathscr{L}_{h}^{n}-\overline{\mathscr{L}}_{h}^{n}\right) \quad$ and $\quad E_{2} \equiv k A\left(\mathscr{L}_{h}^{n}-\overline{\mathscr{L}}_{h}^{n}\right)\left[I+k A \mathscr{L}_{h}^{n}\right]^{-1}$, so that

$$
\begin{aligned}
& \left(\mathscr{L}_{h}^{n}\right)^{1 / 2}\left[I+k A \overline{\mathscr{L}}_{h}^{n}\right]\left(\mathscr{T}_{h}^{n}\right)^{1 / 2}=\left[I+k A \mathscr{L}_{h}^{n}\right]\left[I-\left(\mathscr{L}_{h}^{n}\right)^{1 / 2} E_{1}\left(\mathscr{T}_{h}^{n}\right)^{1 / 2}\right] \\
& \left(\mathscr{T}_{h}^{n}\right)^{1 / 2}\left[I+k A \overline{\mathscr{L}}_{h}^{n}\right]\left(\mathscr{L}_{h}^{n}\right)^{1 / 2}=\left[I-\left(\mathscr{T}_{h}^{n}\right)^{1 / 2} E_{2}\left(\mathscr{L}_{h}^{n}\right)^{1 / 2}\right]\left[I+k A \mathscr{L}_{h}^{n}\right]
\end{aligned}
$$

By (3.1) and (2.8),

$$
\left\|\left(\mathscr{L}_{h}^{n}\right)^{1 / 2} E_{1}\left(\mathscr{T}_{h}^{n}\right)^{1 / 2} \mathbf{X}\right\|+\left\|\left(\mathscr{T}_{h}^{n}\right)^{1 / 2} E_{2}\left(\mathscr{L}_{h}^{n}\right)^{1 / 2} \mathbf{X}\right\| \leq c k\|\mathbf{X}\| \quad \forall \mathbf{X} \in \mathbf{S}_{h} .
$$

Hence, for $k$ small enough, $\left[I+k A \overline{\mathscr{L}}_{h}^{n}\right]$ is invertible. Next, for $\theta_{2}=0, \pm \frac{1}{2}$,

$$
\begin{aligned}
& \left(k \mathscr{L}_{h}^{n}\right)^{1 / 2}\left[I+k A \overline{\mathscr{L}}_{h}^{n}\right]^{-1}\left(k \mathscr{L}_{h}^{n}\right)^{\theta_{2}} \\
& \quad=\left[I-\left(\mathscr{L}_{h}^{n}\right)^{1 / 2} E_{1}\left(\mathscr{T}_{h}^{n}\right)^{1 / 2}\right]^{-1}\left(k \mathscr{L}_{h}^{n}\right)^{\theta_{2}+1 / 2}\left[I+k A \mathscr{L}_{h}^{n}\right]^{-1}
\end{aligned}
$$


and (3.2) follows for $\theta_{1}=\frac{1}{2}$. For $\theta_{1}=0, \pm \frac{1}{2}$

$$
\begin{aligned}
& \left(k \mathscr{L}_{h}^{n}\right)^{\theta_{1}}\left[I+k A \overline{\mathscr{L}}_{h}^{n}\right]^{-1}\left(k \mathscr{L}_{h}^{n}\right)^{1 / 2} \\
& \quad=\left(k \mathscr{L}_{h}^{n}\right)^{\theta_{1}+1 / 2}\left[I+k A \mathscr{L}_{h}^{n}\right]^{-1}\left[I-\left(\mathscr{T}_{h}^{n}\right)^{1 / 2} E_{2}\left(\mathscr{L}_{h}^{n}\right)^{1 / 2}\right]^{-1}
\end{aligned}
$$

and (3.2) follows for $\theta_{2}=\frac{1}{2}$. Now, for the case $\theta_{1}=\theta_{2}=0$, with $\mathbf{X} \in \mathbf{S}_{h}$ chosen arbitrarily,

$$
\|\mathbf{X}\|^{2} \leq \frac{1}{2}\left\|\left[I+k A \overline{\mathscr{L}}_{h}^{n}\right] \mathbf{X}\right\|^{2}+\frac{1}{2}\|\mathbf{X}\|^{2}+k\left|\left(A \overline{\mathscr{L}}_{h}^{n} \mathbf{X}, \mathbf{X}\right)\right| .
$$

Then, with $\boldsymbol{\Psi} \equiv\left(\mathscr{L}_{h}^{n}\right)^{1 / 2} \mathbf{X}$, by $(2.8)$,

$$
\begin{aligned}
\left|\left(A \overline{\mathscr{L}}_{h}^{n} \mathbf{X}, \mathbf{X}\right)\right| & \leq|(A \boldsymbol{\Psi}, \mathbf{\Psi})|+\left|\left(\left(\mathscr{T}_{h}^{n}\right)^{1 / 2}\left[\overline{\mathscr{L}}_{h}^{n}-\mathscr{L}_{h}^{n}\right]\left(\mathscr{T}_{h}^{n}\right)^{1 / 2} \mathbf{\Psi}, A^{T} \mathbf{\Psi}\right)\right| \\
& \leq c(1+k)\left(\mathscr{L}_{h}^{n} \mathbf{X}, \mathbf{X}\right) .
\end{aligned}
$$

Also by (3.2) with $\theta_{1}=\frac{1}{2}, \theta_{2}=0$,

$$
\left\|\left(k \mathscr{L}_{h}^{n}\right)^{1 / 2} \mathbf{X}\right\| \leq c\left\|\left[I+k A \overline{\mathscr{L}}_{h}^{n}\right] \mathbf{X}\right\|,
$$

and the remaining case for (3.2) follows after combining the last three inequalities.

Now, for the sequel, let the following be defined:

$$
\begin{aligned}
& \xi^{n} \equiv U_{h}^{n}-\omega^{n} \text {, } \\
& \eta^{n} \equiv u^{n}-\omega^{n} \\
& r_{h}^{n} \equiv I-k b^{T} \mathscr{L}_{h}^{n}\left[I+k A \mathscr{L}_{h}^{n}\right]^{-1} e \text {, } \\
& \mathscr{R}_{h}^{n} \equiv I-k b^{T} \overline{\mathscr{L}}_{h}^{n}\left[I+k A \overline{\mathscr{L}}_{h}^{n}\right]^{-1} e, \\
& \bar{u}^{n}(s) \equiv \sum_{l=0}^{\mu-1} D^{l} e \partial_{t}^{l} u^{n} \frac{s^{l}}{l !} \quad(0 \leq s \leq k), \quad \bar{u}^{n} \equiv \bar{u}^{n}(k), \\
& \bar{\omega}^{n}(s) \equiv \overline{\mathscr{T}}_{h}^{n}(s) \overline{\mathscr{L}}^{n}(s) \bar{u}^{n}(s) \quad(0 \leq s \leq k), \quad \bar{\omega}^{n} \equiv \bar{\omega}^{n}(k) \text {. }
\end{aligned}
$$

After some straightforward calculations, the following error equation is established:

$$
\begin{aligned}
\xi^{n+1}= & \mathscr{R}_{h}^{n} \xi^{n}+k b^{T} \overline{\mathscr{L}}_{h}^{n}\left[I+k A \overline{\mathscr{L}}_{h}^{n}\right]^{-1}\left\{\bar{\omega}^{n}-e \omega^{n}-k A \sum_{m=0}^{\mu-1} \Gamma_{m}^{n} e \partial_{t} \omega\left(\tau_{m}^{n}\right)\right\} \\
& -k b^{T} A^{-1}\left[I+k A \overline{\mathscr{L}}_{h}^{n}\right]^{-1} A \mathscr{P}_{0} \sum_{m=0}^{\mu-1} \Gamma_{m}^{n} \mathscr{L}\left(\tau_{m}^{n}\right)\left[\bar{u}^{n}-e u\left(\tau_{m}^{n}\right)\right] \\
& -k b^{T} A^{-1}\left[I+k A \overline{\mathscr{L}}_{h}^{n}\right]^{-1} A \sum_{m=n}^{\mu-1} \Gamma_{m}^{n} e \partial_{t}\left\{\left[P_{E}\left(\tau_{m}^{n}\right)-P_{0}\right] u\left(\tau_{m}^{n}\right)\right\} \\
& -\left\{\omega^{n+1}-\omega^{n}-k b^{T} \sum_{m=0}^{\mu-1} \Gamma_{m}^{n} e \partial_{t} \omega\left(\tau_{m}^{n}\right)\right\} \\
& -\left\{\mathscr{R}_{h}^{n} \xi^{n}+\sum_{l=1}^{4} \psi_{l}^{n} \equiv \mathscr{R}_{h}^{n} \xi^{n}+\psi^{n}, \quad 0 \leq n \leq n^{*}-1 .\right.
\end{aligned}
$$

Now, since it is required in Section 4, stability and consistency are established in the following norms, which according to (1.7) are well defined for $0 \leq n \leq n^{*}$ :

$$
\|\chi\|_{n} \equiv\left\{(\chi, \chi)+k\left(L_{h}^{n} \chi, \chi\right)\right\}^{1 / 2}, \quad \chi \in S_{h} .
$$

Also, from (1.10) with $l=0$, it follows that these norms are equivalent:

$$
c_{1}\|\chi\|_{m} \leq\|\chi\|_{n} \leq c_{2}\|\chi\|_{m} \quad \forall \chi \in S_{h}, 0 \leq m, n \leq n^{*} .
$$

As in Section 2, for $\mathbf{X} \in \mathbf{S}_{h}$, take $\|\mathbf{X}\|_{n} \equiv\left\{\sum_{i=1}^{q}\left\|\chi_{i}\right\|_{n}^{2}\right\}^{1 / 2}$. 
In the next two propositions, two classes of IRKM's are considered in order to obtain the following stability inequality for (3.3):

$$
\left\|\xi^{n+1}\right\|_{n+1}^{2} \leq(1+\tilde{c} k)\left\|\xi^{n}\right\|_{n}^{2}+c k^{-1}\left\|\psi^{n}\right\|_{n}^{2}, \quad 0 \leq n \leq n^{*}-1 .
$$

PROPOSITION 3.1. Suppose $\xi^{n+1}=\mathscr{R}_{h}^{n} \xi^{n}+\psi^{n}$, and let (1.19) be satisfied. Then there is a constant $\tilde{c}$ such that (3.5) holds. In fact, $\tilde{c}<0$ if $(1.20)$ holds and c(1) of (1.8) is small enough.

Proof. Triangulating with $r_{h}^{n} \xi^{n}$, one obtains

$$
\begin{aligned}
\left\|\xi^{n+1}\right\|_{n}^{2} & \leq\left\{\left\|\left[\mathscr{R}_{h}^{n}-r_{h}^{n}\right] \xi^{n}\right\|_{n}+\left\|r_{h}^{n} \xi^{n}\right\|_{n}+\left\|\psi^{n}\right\|_{n}\right\}\left\|\xi^{n+1}\right\|_{n} \\
& \leq \frac{1}{2}\left\|r_{h}^{n} \xi^{n}\right\|_{n}^{2}+\frac{1}{2}\left\|\xi^{n+1}\right\|_{n}^{2}(1+\varepsilon k)+c(\varepsilon k)^{-1}\left\{\left\|\left[\mathscr{R}_{h}^{n}-r_{h}^{n}\right] \xi^{n}\right\|_{n}^{2}+\left\|\psi^{n}\right\|_{n}^{2}\right\} .
\end{aligned}
$$

By some straightforward calculations,

$$
\begin{aligned}
\mathscr{R}_{h}^{n}-r_{h}^{n}= & b^{T} A^{-1}\left[I+k A \overline{\mathscr{L}}_{h}^{n}\right]^{-1}\left(k \mathscr{L}_{h}^{n}\right)^{1 / 2} \\
& \times A\left(\mathscr{T}_{h}^{n}\right)^{1 / 2}\left(\mathscr{L}_{h}^{n}-\overline{\mathscr{L}}_{h}^{n}\right)\left(\mathscr{T}_{h}^{n}\right)^{1 / 2}\left(k \mathscr{L}_{h}^{n}\right)^{1 / 2}\left[I+k A \mathscr{L}_{h}^{n}\right]^{-1} e,
\end{aligned}
$$

so that with (2.8), (3.1), and (3.2), it follows that for $\theta_{1}, \theta_{2}=0, \pm \frac{1}{2}$

$$
\left\|\left(k L_{h}^{n}\right)^{\theta_{1}}\left[\mathscr{R}_{h}^{n}-r_{h}^{n}\right]\left(k L_{h}^{n}\right)^{\theta_{2}} \chi\right\| \leq c k\|\chi\| \quad \forall \chi \in S_{h} .
$$

So, there is a $c_{1}>0$ such that

$$
(1-\varepsilon k)\left\|\xi^{n+1}\right\|_{n}^{2} \leq\left\|r_{h}^{n} \xi^{n}\right\|_{n}^{2}+\varepsilon^{-1} c_{1} k\left\|\xi^{n}\right\|^{2}+c k^{-1}\left\|\psi^{n}\right\|_{n}^{2} .
$$

Since (3.6) depends on (2.8) and hence (1.8), the smallness of $c_{1}$ is determined by that of $c(1)$ in (1.8). Now by (2.7), with $\chi^{n+1} \equiv\left(L_{h}^{n}\right)^{1 / 2} \xi^{n+1}$,

$$
\begin{aligned}
\left\|\xi^{n+1}\right\|_{n+1}^{2} & =\left\|\xi^{n+1}\right\|_{n}^{2}+k\left(\left(T_{h}^{n}\right)^{1 / 2}\left[L_{h}^{n+1}-L_{h}^{n}\right]\left(T_{h}^{n}\right)^{1 / 2} \chi^{n+1}, \chi^{n+1}\right) \\
& \leq\left(1+c_{2} k\right)\left\|\xi^{n+1}\right\|_{n}^{2},
\end{aligned}
$$

where again the smallness of $c_{2}$ is determined by that of $c(1)$ in (1.8). Hence, according to the above, there is a $c_{3}>0$ such that

$$
\left(1-c_{3} k\right)\left\|\xi^{n+1}\right\|_{n+1}^{2} \leq\left\|r_{h}^{n} \xi^{n}\right\|_{n}^{2}+\varepsilon^{-1} c_{1} k\left\|\xi_{n}\right\|^{2}+c k^{-1}\left\|\psi^{n}\right\|_{n}^{2} .
$$

Now, since $\nu \geq 1, r(0)=1=-r^{\prime}(0)$. So let $z_{1}, \theta>0$ be chosen so that $|r(z)| \leq$ $1-\theta z, 0 \leq z \leq z_{1}$. By (1.7), $\sigma\left(k L_{h}^{n}\right) \subset\left[\theta^{-1} c_{0} k, \infty\right)$ for some $c_{0}>0$. Thus, using a spectral argument, it follows that for $k$ small enough, there is a $\hat{c} \in\left[-c_{0}, 0\right]$ such that

$$
\left\|r_{h}^{n} \chi\right\| \leq(1+\hat{c} k)\|\chi\| \quad \forall \chi \in S_{h}
$$

and $\hat{c}<0$ if (1.20) holds. Finally, (3.5) follows with (3.7) and (3.8), and $\tilde{c}>$ $\hat{c}+c_{3}+\varepsilon^{-1} c_{1}$.

The conditions of the following proposition guarantee that $\tilde{c}<0$ in (3.5), and hence the approximations decay.

PROPOSITION 3.2. Suppose $\xi^{n+1}=\mathscr{R}_{h}^{n} \xi^{n}+\psi^{n}$ and let (1.20) and (1.21) be satisfied. Then there is a constant $\tilde{c}<0$ such that (3.5) holds.

Proof. Suppose it has been established that for some $\varepsilon_{1}>0$ and $\tilde{c}<0$

$$
\left\|\mathscr{R}_{h}^{n} \xi^{n}\right\|^{2}+\left(1+\varepsilon_{1}\right)\left\|\left(k L_{h}^{n+1}\right)^{1 / 2} \mathscr{R}_{h}^{n} \xi^{n}\right\|^{2} \leq(1+\tilde{c} k)\left\|\xi^{n}\right\|_{n}^{2} .
$$


By (1.7) and (3.4),

$$
\begin{aligned}
\left(\xi^{n+1},\right. & \left.\xi^{n+1}\right)+\left(1+\varepsilon_{1}\right)\left(k L_{h}^{n+1} \xi^{n+1}, \xi^{n+1}\right) \\
= & \left(\mathscr{R}_{h}^{n} \xi^{n}, \xi^{n+1}\right)+\left(1+\varepsilon_{1}\right)\left(\left(k L_{h}^{n+1}\right)^{1 / 2} \mathscr{R}_{h}^{n} \xi^{n},\left(k L_{h}^{n+1}\right)^{1 / 2} \xi^{n+1}\right) \\
& +\left(\left(k L_{h}^{n+1}\right)^{-1 / 2} \psi^{n},\left(k L_{h}^{n+1}\right)^{1 / 2} \xi^{n+1}\right) \\
& +\left(1+\varepsilon_{1}\right)\left(\left(k L_{h}^{n+1}\right)^{1 / 2} \psi^{n},\left(k L_{n}^{n+1}\right)^{1 / 2} \xi^{n+1}\right) \\
\leq & \frac{1}{2}\left\{\left\|\mathscr{R}_{h}^{n} \xi^{n}\right\|^{2}+\left(1+\varepsilon_{1}\right)\left\|\left(k L_{h}^{n+1}\right)^{1 / 2} \mathscr{R}_{h}^{n} \xi^{n}\right\|^{2}\right\} \\
& +\frac{1}{2}\left\{\left\|\xi^{n+1}\right\|^{2}+\left(1+\varepsilon_{1}\right)\left\|\left(k L_{h}^{n+1}\right)^{1 / 2} \xi^{n+1}\right\|^{2}\right\} \\
& +c \varepsilon_{1}^{-1} k^{-1}\left\|\psi^{n}\right\|_{n}^{2}+\frac{1}{2} \varepsilon_{1}\left\|\left(k L_{h}^{n+1}\right)^{1 / 2} \xi^{n+1}\right\|^{2},
\end{aligned}
$$

and (3.5) follows after combining the above inequalities. Now for (3.9), the following are required:

$$
\begin{gathered}
\left\|\mathscr{R}_{h}^{n} \xi^{n}\right\|^{2} \leq\left\|\xi^{n}\right\|^{2}-c\left\|\left(k \mathscr{L}_{h}^{n}\right)^{1 / 2} \bar{\xi}^{n}\right\|^{2} \\
\left\|\mathscr{R}_{h}^{n} \xi^{n}\right\|^{2}+c_{2}\left\|\left(k L_{h}^{n}\right)^{1 / 2} \mathscr{R}_{h}^{n} \xi^{n}\right\|^{2} \leq\left\|\xi^{n}\right\|^{2}+c_{1}\left\|\left(k L_{h}^{n}\right)^{1 / 2} \xi^{n}\right\|^{2}, \\
\left\|\left(k L_{h}^{n}\right)^{1 / 2} \mathscr{R}_{h}^{n} \xi^{n}\right\|^{2} \leq\left(1+c_{3} k\right)\left\|\left(k L_{h}^{n}\right)^{1 / 2} \xi^{n}\right\|^{2}
\end{gathered}
$$

where $\bar{\xi}^{n} \equiv\left[I+k A \overline{\mathscr{L}}_{h}^{n}\right]^{-1} e \xi^{n}$ and $c_{2}>c_{1}>0$. For $(3.10)$, note that $\mathscr{R}_{h}^{n} \xi^{n}=$ $\xi^{n}-k b^{T} \overline{\mathscr{L}}_{h}^{n} \bar{\xi}^{n}$, and hence

$$
\begin{aligned}
\left\|\mathscr{R}_{h}^{n} \xi^{n}\right\|^{2} & =\left\|\xi^{n}\right\|^{2}-2 k\left(e \xi^{n}, B \overline{\mathscr{L}}_{h}^{n} \bar{\xi}^{n}\right)+k^{2}\left(b^{T} \overline{\mathscr{L}}_{h}^{n} \bar{\xi}^{n}, b^{T} \overline{\mathscr{L}}_{h}^{n} \bar{\xi}^{n}\right) \\
& =\left\|\xi^{n}\right\|^{2}-2 k\left(\left[I+k A \overline{\mathscr{L}}_{h}^{n}\right] \bar{\xi}^{n}, B \overline{\mathscr{L}}_{h}^{n} \bar{\xi}^{n}\right)+k^{2}\left(b b^{T} \overline{\mathscr{L}}_{h}^{n} \bar{\xi}^{n}, \overline{\mathscr{L}}_{h}^{n} \bar{\xi}^{n}\right) \\
& =\left\|\xi^{n}\right\|^{2}-2 k\left(B \bar{\xi}^{n}, \overline{\mathscr{L}}_{h}^{n} \bar{\xi}^{n}\right)-k^{2}\left(M \overline{\mathscr{L}}_{h}^{n} \bar{\xi}^{n}, \overline{\mathscr{L}}_{h}^{n} \bar{\xi}^{n}\right) .
\end{aligned}
$$

The last term can be ignored since by $(1.21), M$ is positive semidefinite. Also, for $\mathbf{X} \in \mathbf{S}_{h}$ and $\boldsymbol{\Psi}=\left(\mathscr{L}_{h}^{n}\right)^{1 / 2} \mathbf{X}$, by (1.21) and (2.8),

$$
\begin{aligned}
\left(B \mathbf{X}, \overline{\mathscr{L}}_{h}^{n} \mathbf{X}\right) & =\left\|B^{1 / 2} \Psi\right\|^{2}+\left(B \Psi,\left(\mathscr{T}_{h}^{n}\right)^{1 / 2}\left[\overline{\mathscr{L}}_{h}^{n}-\mathscr{L}_{h}^{n}\right]\left(\mathscr{T}_{h}^{n}\right)^{1 / 2} \boldsymbol{\Psi}\right) \\
& \leq c(1-k)\left\|\left(\mathscr{L}_{h}^{n}\right)^{1 / 2} \mathbf{X}\right\|^{2} .
\end{aligned}
$$

Hence, (3.10) is established. Next, for (3.11), write $\mathscr{R}_{h}^{n} \xi^{n}=\left(I-b^{T}-A^{-1} e\right) \xi^{n}+$ $b^{T} A^{-1} \bar{\xi}^{n}$ and note that by (1.18) and (1.20), $r(\infty) \equiv 1-b^{T} A^{-1} e \in(-1,1)$. So, using (3.10),

$$
\begin{aligned}
\left\|\left(L_{h}^{n}\right)^{1 / 2} \mathscr{R}_{h}^{n} \xi^{n}\right\|^{2}= & r(\infty)\left(\left(L_{h}^{n}\right)^{1 / 2} \mathscr{R}_{h}^{n} \xi^{n},\left(L_{h}^{n}\right)^{1 / 2} \xi^{n}\right) \\
& +\left(\left(L_{h}^{n}\right)^{1 / 2} \mathscr{R}_{h}^{n} \xi^{n}, b^{T} A^{-1}\left(\mathscr{L}_{h}^{n}\right)^{1 / 2} \bar{\xi}^{n}\right) \\
\leq & \frac{1}{2}|r(\infty)|\left\{\left\|\left(L_{h}^{n}\right)^{1 / 2} \mathscr{R}_{h}^{n} \xi^{n}\right\|^{2}+\left\|\left(L_{h}^{n}\right)^{1 / 2} \xi^{n}\right\|^{2}\right\} \\
& +\varepsilon_{2}\left\|\left(L_{h}^{n}\right)^{1 / 2} \mathscr{R}_{h}^{n} \xi^{n}\right\|^{2}+c_{4} \varepsilon_{2}^{-1} k^{-1}\left\{\left\|\xi^{n}\right\|^{2}-\left\|\mathscr{R}_{h}^{n} \xi^{n}\right\|^{2}\right\},
\end{aligned}
$$

and (3.11) follows if $\varepsilon_{2}>0$ is chosen so that $c_{2} \equiv\left(1-\varepsilon_{2}-\frac{1}{2}|r(\infty)|\right) \varepsilon_{2} c_{4}^{-1}>$ $\frac{1}{2}|r(\infty)| \varepsilon_{2} c_{4}^{-1} \equiv c_{1}$. Finally, (3.12) follows after using (3.6) and (1.19) in

$$
\begin{aligned}
\left\|\left(k L_{h}^{n}\right)^{1 / 2} \mathscr{R}_{h}^{n} \xi^{n}\right\| \leq & \left\|\left[\left(k L_{h}^{n}\right)^{1 / 2}\left(\mathscr{R}_{h}^{n}-r_{h}^{n}\right)\left(k L_{h}^{n}\right)^{-1 / 2}\right]\left(k L_{h}^{n}\right)^{1 / 2} \xi^{n}\right\| \\
& +\left\|r_{h}^{n}\left(k L_{h}^{n}\right)^{1 / 2} \xi^{n}\right\| .
\end{aligned}
$$

Next, (3.11) and (3.12) are used to obtain (3.9). Suppose that $\varepsilon_{2}$ is small enough that $c_{2}<1$. Then, assume that $k_{0}>0$ is small enough that if $\theta \equiv\left(1-c_{1}\right) /\left(1+c_{3} k_{0}\right)$, 
then $c_{2}+\theta>1$. Next, multiply (3.12) by $\theta$ and add the result to (3.11). With $c_{5} \equiv c_{2}+\theta-1>0$, and $0<k \leq k_{0}$,

$$
\left\|\mathscr{R}_{h}^{n} \xi^{n}\right\|^{2}+\left(1+c_{5}\right)\left\|\left(k L_{h}^{n}\right)^{1 / 2} \mathscr{R}_{h}^{n} \xi^{n}\right\|^{2} \leq\left\|\xi^{n}\right\|_{n}^{2} .
$$

By (1.7), there is a $c_{6}>0$ such that

$$
c_{6} k\left\|\mathscr{R}_{h}^{n} \xi^{n}\right\|^{2} \leq \frac{1}{2} c_{5}\left\|\left(k L_{h}^{n}\right)^{1 / 2} \mathscr{R}_{h}^{n} \xi^{n}\right\|^{2} .
$$

Also, by (2.7), with $\chi^{n} \equiv\left(k L_{h}^{n}\right)^{1 / 2} \mathscr{R}_{h}^{n} \xi^{n}$,

$$
\begin{aligned}
\left\|\left(k L_{h}^{n+1}\right)^{1 / 2} \mathscr{R}_{h}^{n} \xi^{n}\right\|^{2} & =\left\|\chi^{n}\right\|^{2}+\left(\left(T_{h}^{n}\right)^{1 / 2}\left[L_{h}^{n+1}-L_{h}^{n}\right]\left(T_{h}^{n}\right)^{1 / 2} \chi^{n}, \chi^{n}\right) \\
& \leq\left(1+c_{7} k\right)\left\|\left(k L_{h}^{n}\right)^{1 / 2} \mathscr{R}_{h}^{n} \xi^{n}\right\|^{2} .
\end{aligned}
$$

From the last three inequalities it follows that

$$
\left(1+c_{6} k\right)\left\|\mathscr{R}_{h}^{n} \xi^{n}\right\|^{2}+\left(1+\frac{1}{2} c_{5}\right)\left(1+c_{7} k\right)^{-1}\left\|\left(k L_{h}^{n+1}\right)^{1 / 2} \mathscr{R}_{h}^{n} \xi^{n}\right\|^{2} \leq\left\|\xi^{n}\right\|_{n}^{2} .
$$

So assume that $k_{0}$ above is also small enough that

$$
\left(1+\frac{1}{2} c_{5}\right)\left(1+c_{6} k_{0}\right)^{-1}\left(1+c_{7} k_{0}\right)^{-1} \geq 1+\varepsilon_{1}
$$

for some $\varepsilon_{1}>0$. Then (3.9) follows for some $\tilde{c} \in\left(-c_{6}, 0\right)$.

The next two lemmas are useful in subsequent consistency estimates.

LEMMA 3.2. Fix integers $l, m \geq 0$, and let $t_{0}, t_{1}, t_{2} \in\left[0, t^{*}\right]$ with $\left|t_{2}-t_{1}\right| \leq c k$. Then

$$
\sup _{0 \leq t \leq t^{*}}\left\|L_{h}^{\theta}\left(t_{0}\right) \partial_{s}^{l} \omega(t)\right\| \leq c(l)\left\|u^{0}\right\|_{2(l+1)}, \quad \theta=0, \frac{1}{2} .
$$

Also, with $E \equiv \int_{t_{1}}^{t_{2}}\left(t_{2}-t\right)^{m} \partial_{t}^{l} \omega(t) d t$, there exist $E_{1}$ and $E_{2}$ such that $E=E_{1}+E_{2}$ while

$$
\begin{gathered}
\left\|\left[k L_{h}\left(t_{0}\right)\right]^{1 / 2} E_{1}\right\| \leq c(l) h k^{m+1 / 2+i}\left\|u^{0}\right\|_{2(l+i)}, \quad i=0,1 \\
\left\|k L_{h}\left(t_{0}\right) E_{2}\right\| \leq c(l) k^{m+1+i}\left\|u^{0}\right\|_{2(l+i)}, \quad i=0,1 .
\end{gathered}
$$

Furthermore, for $0 \leq n \leq n^{*}$,

$$
\|E\|_{n} \leq c(l)\left(k^{m+1}+h k^{m+1 / 2}+h^{2} k^{m}\right)\left\|u^{0}\right\|_{2 l} .
$$

Proof. See [14].

LEMMA 3.3. The following holds for $0 \leq s \leq k$ and $0 \leq t \leq t^{*}$ :

$$
\left\|\partial_{s}^{\mu} \bar{\omega}^{n}(s)\right\|+h\left\|\mathscr{L}_{h}^{1 / 2}(t) \partial_{s}^{\mu} \bar{\omega}^{n}(s)\right\| \leq c h^{2}\left\|u^{0}\right\|_{2 \mu} .
$$

Proof. See [14].

The order of consistency is established as follows.

PROPOSITION 3.3. The term $\psi^{n}$ in (3.3) satisfies

$$
\left\|\psi^{n}\right\|_{n} \leq c k\left(h^{r}+k^{\mu}+h k^{\mu-1 / 2}+h^{2} k^{\mu-1}\right)\left\|u^{0}\right\|_{2(\mu+1)} .
$$

Proof. First, consider $\psi_{1}^{n}$. By differentiating $\overline{\mathscr{L}}_{h}^{n}(s) \bar{\omega}^{n}(s)=\mathscr{P}_{0} \overline{\mathscr{L}}^{n}(s) \bar{u}^{n}(s)$ and using (1.28), it can be shown inductively that $\partial_{s}^{l} \bar{\omega}^{n}(0)=D^{l} e \partial_{t}^{l} \omega^{n}, 0 \leq l \leq \mu-1$. Therefore,

$$
\bar{\omega}^{n}-e \omega^{n}=\sum_{l=1}^{\mu-1} D^{l} e \partial_{t}^{l} \omega^{n} \frac{k^{l}}{l !}+E, \quad E \equiv \frac{1}{(\mu-1) !} \int_{0}^{k}(k-s)^{\mu-1} \partial_{s}^{\mu} \bar{\omega}^{n}(s) d s .
$$


Next, by (1.28) and (1.26),

$$
\begin{gathered}
k A \sum_{m=0}^{\mu-1} \Gamma_{m}^{n} e \partial_{t} \omega\left(\tau_{m}^{n}\right)=\sum_{l=1}^{\mu-1} D^{l} e \partial_{t}^{l} \omega^{n} \frac{k^{l}}{l !}+F \\
F \equiv \frac{k}{(\mu-2) !} \sum_{m=0}^{\mu-1} A \Gamma_{m}^{n} e \int_{t^{n}}^{\tau_{m}^{n}}\left(\tau_{m}^{n}-t\right)^{\mu-2} \partial_{t}^{\mu} \omega(t) d t .
\end{gathered}
$$

Now $\psi_{1}^{n}=\tilde{E}-\tilde{F}$, with

$$
\begin{aligned}
\tilde{E} \equiv & k^{1 / 2} b^{T} A^{-1}\left[I+k A \overline{\mathscr{L}}_{h}^{n}\right]^{-1}\left(k \mathscr{L}_{h}^{n}\right)^{1 / 2} A\left(\mathscr{T}_{h}^{n}\right)^{1 / 2} \overline{\mathscr{L}}_{h}^{n}\left(\mathscr{T}_{h}^{n}\right)^{1 / 2}\left[\left(\mathscr{L}_{h}^{n}\right)^{1 / 2} E\right], \\
\tilde{F} \equiv & b^{T} A^{-1}\left[I+k A \overline{\mathscr{L}}_{h}^{n}\right]^{-1}\left(k \mathscr{L}_{h}^{n}\right)^{1 / 2} A\left(\mathscr{T}_{h}^{n}\right)^{1 / 2}\left[\overline{\mathscr{L}}_{h}^{n}-\mathscr{L}_{h}^{n}\right]\left(\mathscr{T}_{h}^{n}\right)^{1 / 2}\left[\left(k \mathscr{L}_{h}^{n}\right)^{1 / 2} F\right] \\
& +b^{T} A^{-1}\left[I+k A \overline{\mathscr{L}}_{h}^{n}\right]^{-1}\left(k \mathscr{L}_{h}^{n}\right)^{1 / 2} A\left[\left(k \mathscr{L}_{h}^{n}\right)^{1 / 2} F_{1}\right] \\
& +b^{T} A^{-1}\left[I+k A \overline{\mathscr{L}}_{h}^{n}\right]^{-1} A\left[k \mathscr{L}_{h}^{n} F_{2}\right],
\end{aligned}
$$

where $F=F_{1}+F_{2}$ is the splitting guaranteed by Lemma 3.2. The estimate required for $\psi_{1}^{n}$ then follows using (3.2), (2.6) (3.17), (1.29), (2.8), and inequalities of the form (3.14)-(3.16). Now consider $\psi_{2}^{n}$. After differentiating $\bar{v}(s) \equiv \overline{\mathscr{L}}^{n}(s) \bar{u}^{n}(s)$, with (1.28) and (1.1) it can be shown that $\partial_{s}^{l} \bar{v}(0)=-D^{l} e \partial_{t}^{l+1} u^{n}, 0 \leq l \leq \mu-1$. Hence,

$$
\overline{\mathscr{L}}^{n} \bar{u}^{n}=-\sum_{l=0}^{\mu-1} D^{l} e \partial_{t}^{l+1} u^{n} \frac{k^{l}}{l !}+\frac{1}{(\mu-1) !} \int_{0}^{k}(k-s)^{\mu-1} \partial_{s}^{\mu} \bar{v}(s) d s .
$$

Then, by (1.1) and (1.28),

$$
\begin{aligned}
-\sum_{m=0}^{\mu-1} \Gamma_{m}^{n} \mathscr{L}\left(\tau_{m}^{n}\right) e u\left(\tau_{m}^{n}\right)= & \sum_{l=0}^{\mu-1} D^{l} e \partial_{t}^{l+1} u^{n} \frac{k^{l}}{l !} \\
& +\frac{1}{(\mu-1) !} \sum_{m=0}^{\mu-1} \Gamma_{m}^{n} e \int_{t^{n}}^{\tau_{m}^{n}}\left(\tau_{m}^{n}-t\right)^{\mu-1} \partial_{t}^{\mu+1} u(t) d t .
\end{aligned}
$$

So the required estimate for $\psi_{2}^{n}$ follows with (3.2), (2.2), (1.4), and (1.29). Next, the estimate required for $\psi_{3}^{n}$ follows with (3.2), (1.29), (1.12), (1.13), and (1.4). Finally, consider $\psi_{4}^{n}$. First,

$$
\omega^{n+1}-\omega^{n}=\sum_{l=1}^{\mu} \partial_{t}^{l} \omega^{n} \frac{k^{l}}{l !}+G, \quad G \equiv \frac{1}{\mu !} \int_{t^{n}}^{t^{n+1}}\left(t^{n+1}-t\right)^{\mu} \partial_{t}^{\mu+1} \omega(t) d t .
$$

By (1.28) and (1.27),

$$
\begin{gathered}
k b^{T} \sum_{m=0}^{\mu-1} \Gamma_{m}^{n} e \partial_{t} \omega\left(\tau_{m}^{n}\right)=\sum_{l=1}^{\mu} \partial_{t}^{l} \omega^{n} \frac{k^{l}}{l !}+H, \\
H \equiv \frac{k}{(\mu-1) !} \sum_{m=0}^{\mu-1} b^{T} \Gamma_{m}^{n} e \int_{t^{n}}^{\tau_{m}^{n}}\left(\tau_{m}^{n}-t\right)^{\mu-1} \partial_{t}^{\mu+1} \omega(t) d t
\end{gathered}
$$

Hence, $\psi_{4}^{n}=-G+H$, and the estimate required for $\psi_{4}^{n}$ follows after applying Lemma 3.2 to $G$ and $H$, and using (1.29) to obtain inequalities of the form (3.16). 
With the consistency complete, it is now appropriate to discuss the development of the techniques used. First, it is possible to construct an error equation alternative to (3.3) which circumvents the constructions of Section 2. However, this requires inverse properties. For example, one option involves the following replacements:

$$
\begin{gathered}
\bar{\omega}^{n} \rightarrow \sum_{l=0}^{\mu-1} D^{l} e \partial_{t}^{l} \omega^{n} \frac{k^{l}}{l !} \\
\psi_{2}^{n} \rightarrow k b^{T} A^{-1}\left[I+k A \overline{\mathscr{L}}_{h}^{n}\right]^{-1} A \sum_{m=0}^{\mu-1} \Gamma_{m}^{n} \mathscr{L}_{h}\left(\tau_{m}^{n}\right)\left[\bar{\omega}^{n}-e \omega\left(\tau_{m}^{n}\right)\right] .
\end{gathered}
$$

Then in the above proof, $\bar{v}(s)$ is changed to $\overline{\mathscr{L}}_{h}^{n}(s) \bar{\omega}^{n}(s)$, and bounding derivatives of the latter involves bounding products of the form $L_{h}^{(i)}(s) T_{h}^{(j)}(t)$. This can be accomplished using inverse assumptions as demonstrated by Bales [2].

Also, the original idea for overcoming the suboptimal convergence rates mentioned in connection with (1.33) was to find $q \times q$ matrices $\left\{D_{l}\right\}_{l=0}^{\nu-1}$ with which the following would lead to optimal convergence estimates:

$$
\begin{gathered}
\sum_{m=0}^{\nu-1} \Gamma_{m}^{n}\left(\delta_{m}^{n}\right)^{l} \equiv D_{l}, \quad 0 \leq l \leq \nu-1 ; \\
\overline{\mathscr{L}}_{h}^{n} \equiv \sum_{m=0}^{\nu-1} \Gamma_{m}^{n} \mathscr{L}_{h}\left(t^{n}+\delta_{m}^{n} k\right) \approx \sum_{l=0}^{\nu-1} D_{l} \mathscr{L}_{h}^{(l)}\left(t^{n}\right) \frac{k^{l}}{l !}
\end{gathered}
$$

However, attempts to prove an optimal order of consistency have repeatedly led to the following conditions for the matrices $\left\{D_{l}\right\}_{l=0}^{\nu-1}$ :

$$
\begin{aligned}
& D_{0} e=e ; \quad D_{i} D_{j} e=D_{i+j} e, \quad 0 \leq i, j, i+j \leq \nu-1 ; \\
& l A D_{l-1} e=D_{l} e, \quad 1 \leq l \leq \nu-1 .
\end{aligned}
$$

To see this, consider for example adapting the estimate for $\psi_{1}^{n}$. Unfortunately, even though the number of unknowns matches the number of constraints in the equations above, it is shown in [15] that they can be solved only if $\nu \leq q+1$.

Now, (1.5) is established in the following for (1.32).

THEOREM 3.4. Under the conditions of Lemma 3.1 and either Proposition 3.1 or 3.2, $\left\{U_{h}^{n}\right\}_{n=0}^{n^{*}}$ are well defined by (1.31) and (1.32), and the following holds:

$$
\max _{0 \leq n \leq n^{*}}\left\|U_{h}^{n}-u^{n}\right\| \leq c^{*}\left(h^{r}+k^{\mu}+h k^{\mu-1 / 2}+h^{2} k^{\mu-1}\right)\left\|u^{0}\right\|_{\alpha} .
$$

Also, unless $\tilde{c}<0$ in (3.5), $c^{*}$ depends exponentially on $t^{*}$.

Proof. Set $E \equiv\left[\left(h^{r}+k^{\mu}+h k^{\mu-1 / 2}+h^{2} k^{\mu-1}\right)\left\|u^{0}\right\|_{\alpha}\right]^{2}$. Then, combining (3.5) and (3.18) for (3.3),

$$
\left\|\xi^{n+1}\right\|_{n+1}^{2} \leq(1+\tilde{c} k)\left\|\xi^{n}\right\|_{n}^{2}+c_{1} k E, \quad 0 \leq n \leq n^{*}-1 .
$$

After dividing this by $(1+\tilde{c} k)^{n+1}$ and summing, the result is

$$
\left\|\xi^{n}\right\|_{n}^{2} \leq(1+\tilde{c} k)^{n}\left\|\xi^{0}\right\|_{0}^{2}+c_{1}|\tilde{c}|^{-1}\left|1-(1+\tilde{c} k)^{n}\right| E, \quad 0 \leq n \leq n^{*} .
$$


Now, according to (1.31), $\left[I+k L_{h}^{0}\right] \xi^{0}=\left[P_{0}-P_{E}^{0}\right] u^{0}$. So with (1.13), (1.11), and a spectral argument, it follows that

$$
\left\|\xi^{0}\right\|_{0}^{2} \leq c\left\|\left[P_{0}-P_{E}^{0}\right] u^{0}\right\|\left\|\left[I+k L_{h}\right]^{-1}\left[P_{0}-P_{E}^{0}\right] u^{0}\right\| \leq c h^{r}\left\|u^{0}\right\|_{r} .
$$

Then, (3.19) follows with (1.12) and the last two inequalities.

4. Iterative Approximations. In this section, the iterative scheme (1.39) is analyzed for the approximation of the solution to (1.1), and (1.5) is established. First, a brief discussion of Preconditioned Iterative Methods (PIM's) is given. See Hageman and Young [11] for more information.

Let $H$ be any finite-dimensional Hilbert space equipped with an inner product $(\cdot, \cdot)_{H}$ and an associated norm $\|\cdot\|_{H}$. Also, let $Q: H \rightarrow H$ be $H$-selfadjoint and positive definite, and suppose that an approximation is required for the solution $x^{*}$ to $Q x^{*}=b$. Then, suppose that $Q_{0}: H \rightarrow H$ is $H$-selfadjoint and positive definite, and that solving $Q_{0} \tilde{x}=b$ is relatively inexpensive. Furthermore, assume that $Q$ and $Q_{0}$ are equivalent in the sense that

$$
\rho_{1}\left(Q_{0} x, x\right)_{H} \leq(Q x, x)_{H} \leq \rho_{2}\left(Q_{0} x, x\right)_{H} \quad \forall x \in H .
$$

The operator $Q_{0}$ is called the preconditioner and the PIM's of interest in this work are those with the following properties:

(i) If $\left\{x_{j}\right\}_{j=0}^{J}$ are given approximations to $x^{*}$, the solution of $Q x^{*}=b$, then calculating $x_{J+1}$ only involves computing $Q x, Q_{0} x,(Q x, x)_{H}$, and $\left(Q_{0} x, x\right)_{H}$ for certain $x \in H$, and solving equations of the form $Q_{0} \tilde{x}=b$.

(ii) There is a smooth decreasing function $\sigma:(0,1) \rightarrow(0,1)$ such that $\sigma(1)=0$ and, if (4.1) holds, then

$$
\left\|Q_{0}^{1 / 2}\left[x^{*}-x_{j}\right]\right\|_{H} \leq c\left[\sigma\left(\rho_{1} / \rho_{2}\right)\right]^{j}\left\|Q_{0}^{1 / 2}\left[x^{*}-x_{0}\right]\right\|_{H} .
$$

For example, the Preconditioned Conjugate Gradient Method satisfies the above properties, and it is popular for having $\sigma(s)=(1-\sqrt{s}) /(1+\sqrt{s})$ as opposed to, say, $(1-s) /(1+s)$, which is offered by various other PIM's.

Now, the rough discussion prior to (1.39) is expanded with more details. First, suppose that for $0 \leq n \leq n^{*}-1,\left\{U_{h}^{m}\right\}_{m=0}^{n}$ and $\left\{\tilde{U}_{h}^{m}\right\}_{m=0}^{n-1}$ have been computed using methods described below, and recall that an efficient procedure is needed for computing $\bar{U}_{h}^{n}$ defined by (1.34). Next, let $\tilde{V}_{0}^{n}$ denote an initial approximation to $\bar{U}_{h}^{n}$ given as indicated in (1.36). Now, instead of actually computing $\left\{\bar{V}_{l}^{n}\right\}_{0 \leq l \leq l_{n}}$ as suggested by (1.35), proceed as follows. Let a sequence of positive integers $\left\{j_{n}\right\}_{n=0}^{n^{*}-1}$ be specified. Then, suppose in an inductive fashion, that for $l \geq 1, \tilde{V}_{l-1, j_{n}}^{n}$ has been computed from $j_{n}$ PIM iterations as prescribed below, and let $\tilde{V}_{l}^{n}$ be defined by the outer iteration:

$$
\left[I+k \Lambda \mathscr{L}_{h}^{n}\right]\left(S \tilde{V}_{l}^{n}\right)=\left\{S e U_{h}^{n}+k S A\left(\mathscr{L}_{h}^{n}-\overline{\mathscr{L}}_{h}^{n}\right) \tilde{V}_{l-1, j_{n}}^{n}\right\}, \quad 1 \leq l \leq l_{n},
$$

with the understanding that $\tilde{V}_{0, j_{n}}^{n} \equiv \tilde{V}_{0}^{n}$. Letting $n$ and $l$ be fixed, (4.3) can be written in the form

$$
\left[I+k \lambda_{i} L_{h}^{n}\right] \psi_{i}=\phi_{i}-k \theta_{i} L_{h}^{n} \psi_{i-1}, \quad 1 \leq i \leq q,
$$

where $\psi_{0} \equiv 0, \psi_{i} \equiv\left(S \tilde{V}_{l}^{n}\right)_{i}, 1 \leq i \leq q$, and according to (1.28) with $l=0$,

$$
\phi_{i} \equiv\left[S e U_{h}^{n}+k \Lambda S \sum_{m=1}^{\mu-1} \Gamma_{m}^{n}\left(\mathscr{L}_{h}^{n}-\mathscr{L}_{h}\left(\tau_{m}^{n}\right)\right) \tilde{V}_{l-1, j_{n}}^{n}\right]_{i} \text {. }
$$


The natural preconditioning for (4.4) involves $\left[I+k L_{h}^{0}\right]$ which, according (1.10) and (1.24), is equivalent to the operators of (4.4), i.e., for $1 \leq i \leq q$ and $0 \leq n \leq n^{*}$,

$$
\rho_{1}\left(\left[I+k L_{h}^{0}\right] \chi, \chi\right) \leq\left(\left[I+\lambda_{i} L_{h}^{n}\right] \chi, \chi\right) \leq \rho_{2}\left(\left[I+k L_{h}^{0}\right] \chi, \chi\right) \quad \forall \chi \in S_{h}
$$

Now, to cover the case that $A$ is not diagonalizable, define $\tilde{\psi}_{i}$ with

$$
\left[I+k \lambda_{i} L_{h}^{n}\right] \tilde{\psi}_{i}=\phi_{i}-k \theta_{i} L_{h}^{n} \tilde{\psi}_{i-1}^{j_{n}}, \quad 1 \leq i \leq q
$$

where $\tilde{\psi}_{0}^{j_{n}} \equiv 0$. Also, to obtain $\tilde{\psi}_{i}^{j_{n}}$ for $1 \leq i \leq q$, set $\tilde{\psi}_{i}^{0} \equiv\left(S \tilde{V}_{l-1, j_{n}}^{n}\right)_{i}$ and let iterates $\left\{\tilde{\psi}_{i}^{j}\right\}_{0 \leq j \leq j_{n}}$ be given by a PIM with preconditioner $\left[I+k L_{h}^{0}\right]$. Then as (4.2) follows from (4.1), from (4.6) it follows that

$$
\left\|\tilde{\psi}_{i}-\tilde{\psi}_{i}^{j}\right\|_{0} \leq c\left[\sigma\left(\rho_{1} / \rho_{2}\right)\right]^{j}\left\|\tilde{\psi}_{i}-\tilde{\psi}_{i}^{0}\right\|_{0} .
$$

Finally, take $\Psi \equiv\left\langle\psi_{1}, \psi_{2}, \ldots, \psi_{q}\right\rangle^{T}$ and $\tilde{\Psi}^{j} \equiv\left\langle\tilde{\psi}_{1}^{j}, \tilde{\psi}_{2}^{j}, \ldots, \tilde{\psi}_{q}^{j}\right\rangle^{T}$, so that $\tilde{V}_{l}^{n}=$ $S^{-1} \Psi$ and inner iterates for (4.3) are defined by

$$
\tilde{V}_{l, 0}^{n}=\tilde{V}_{l-1, j_{n}}^{n}, \quad \tilde{V}_{l, j}^{n} \equiv S^{-1} \tilde{\Psi}^{j}, \quad 1 \leq j \leq j_{n} .
$$

It is shown in [14] that

$$
\left\|\bar{U}_{h}^{n}-\tilde{V}_{l, j_{n}}^{n}\right\|_{0} \leq\left(c k+c\left[\sigma\left(\rho_{1} / \rho_{2}\right)\right]^{j_{n}}\right)\left\|\bar{U}_{h}^{n}-\tilde{V}_{l-1, j_{n}}^{n}\right\|_{0}, \quad l \geq 1 .
$$

So, given some $\varepsilon_{0}>0, j_{n}$ is chosen so that

$$
\left\|\bar{U}_{h}^{n}-\tilde{V}_{l, j_{n}}^{n}\right\|_{0} \leq \beta_{n}\left\|\bar{U}_{h}^{n}-\tilde{V}_{l-1, j_{n}}^{n}\right\|_{0}, \quad 1 \leq l \leq l_{n},
$$

where

$$
\beta_{n}^{2} \leq \begin{cases}c k^{2}, & 0 \leq n \leq \mu \\ \varepsilon_{0} t^{n+1}, & 0 \leq n \leq n^{*}-1\end{cases}
$$

Finally, $\tilde{U}_{h}^{n}$ and $U_{h}^{n+1}$ are given by (1.39). From the last three inequalities, it follows that the integers $\left\{j_{n}\right\}_{n=0}^{n^{*}-1}$ may be chosen so that $\sum_{n=0}^{n^{*}-1} l_{n} j_{n} / n^{*} \leq c$ as claimed in the Introduction.

The next objective is to show that the convergence rate (1.5) can be preserved even when $\left\{j_{n}\right\}_{n=0}^{n^{*}-1}$ are chosen so that (4.9) and (4.10) hold. So additional stability and consistency results follow. First, define $\varsigma^{n} \equiv U_{h}^{n}-\omega^{n}$ and $\psi^{n} \equiv \mathscr{R}_{h}^{n} \omega^{n}-\omega^{n+1}$, where $\psi^{n}$ is as in (3.3). Now, according to (1.39) and (1.32), $U_{h}^{n+1}-\mathscr{R}_{h}^{n} U_{h}^{n}=$ $-b^{T} A^{-1}\left(\bar{U}_{h}^{n}-\tilde{U}_{h}^{n}\right)$. Thus,

$$
\varsigma^{n+1}=\mathscr{R}_{h}^{n} \varsigma^{n}+\psi^{n}-b^{T} A^{-1}\left(\bar{U}_{h}^{n}-\tilde{U}_{h}^{n}\right) .
$$

By (4.9) and (1.39), $\left(\bar{U}_{h}^{n}-\tilde{U}_{h}^{n}\right)$ can be estimated in terms of $\left(\bar{U}_{h}^{n}-\tilde{V}_{0}^{n}\right)$. So, the error equation (4.11) is supplemented with the following one, which is obtained 
from (1.34) and (1.36) after some straightforward calculations:

$$
\begin{aligned}
& \bar{U}_{h}^{n}-\tilde{V}_{0}^{n} \\
& =\sum_{m=n-1-\mu_{n}}^{n-1}(-1)^{n-m}\left(\begin{array}{c}
\mu_{n}+1 \\
n-m
\end{array}\right)\left[I+k A \overline{\mathscr{L}}_{h}^{n}\right]^{-1} k A\left(\overline{\mathscr{L}}_{h}^{n}-\overline{\mathscr{L}}_{h}^{m}\right) \\
& \times\left[I+k A \overline{\mathscr{L}}_{h}^{m}\right]^{-1} e \varsigma^{m} \\
& +\left[I+k A \overline{\mathscr{L}}_{h}^{n}\right]^{-1} \sum_{m=n-1-\mu_{n}}^{n-1}(-1)^{n-m-1}\left(\begin{array}{c}
\mu_{n} \\
n-m-1
\end{array}\right)\left[\varsigma^{m+1}-\varsigma^{m}\right] \\
& -\sum_{m=n-1-\mu_{n}}^{n-1}(-1)^{n-m}\left(\begin{array}{c}
\mu_{n}+1 \\
n-m
\end{array}\right)\left(\bar{U}_{h}^{m}-\tilde{U}_{h}^{m}\right) \\
& -\sum_{m=n-1-\mu_{n}}^{n-1}(-1)^{n-m}\left(\begin{array}{c}
\mu_{n}+1 \\
n-m
\end{array}\right)\left[I+k A \overline{\mathscr{L}}_{h}^{n}\right]^{-1} k A\left(\overline{\mathscr{L}}_{h}^{n}-\overline{\mathscr{L}}_{h}^{m}\right) \\
& \times\left[I+k A \overline{\mathscr{L}}_{h}^{m}\right]^{-1}\left\{\bar{\omega}^{m}-e \omega^{m}-k A \sum_{i=0}^{\mu-1} \Gamma_{i}^{m} e \partial_{t} \omega\left(\tau_{i}^{m}\right)\right\} \\
& -k \sum_{m=n-1-\mu_{n}}^{n}(-1)^{n-m}\left(\begin{array}{c}
\mu_{n}+1 \\
n-m
\end{array}\right)\left[I+k A \overline{\mathscr{L}}_{h}^{m}\right]^{-1} A \mathscr{P}_{0} \\
& \times \sum_{i=0}^{\mu-1} \Gamma_{i}^{m} \mathscr{L}\left(\tau_{i}^{m}\right)\left[\bar{u}^{m}-e u\left(\tau_{i}^{m}\right)\right] \\
& -k \sum_{m=n-1-\mu_{n}}^{n}(-1)^{n-m}\left(\begin{array}{c}
\mu_{n}+1 \\
n-m
\end{array}\right)\left[I+k A \overline{\mathscr{L}}_{h}^{m}\right]^{-1} \mathscr{P}_{0} \\
& \times \sum_{i=0}^{\mu-1} \Gamma_{i}^{m} \partial_{t}\left\{\left[P_{E}\left(\tau_{i}^{m}\right)-P_{0}\right] u\left(\tau_{i}^{m}\right)\right\} \\
& +\sum_{m=n-1-\mu_{n}}^{n}(-1)^{n-m}\left(\begin{array}{c}
\mu_{n}+1 \\
n-m
\end{array}\right) \bar{\omega}^{m} \\
& -\left[I+k A \overline{\mathscr{L}}_{h}^{n}\right]^{-1} \sum_{m=n-1-\mu_{n}}^{n}(-1)^{n-m}\left(\begin{array}{c}
\mu_{n}+1 \\
n-m
\end{array}\right) \\
& \times\left\{\bar{\omega}^{m}-e \omega^{m}-k A \sum_{i=0}^{\mu-1} \Gamma_{i}^{m} e \partial_{t} \omega\left(\tau_{i}^{m}\right)\right\} .
\end{aligned}
$$

Before analyzing these error equations, a few adjustments must be made in Propositions 3.1 and 3.2 for the following stronger stability inequality:

$$
\begin{aligned}
\left\|s^{n+1}\right\|_{n+1}^{2} \leq & (1+\tilde{c} k)\left\|s^{n}\right\|_{n}^{2}-c_{0}\left\|\left[I-r_{h}^{n}\right]^{1 / 2} \varsigma^{n}\right\|_{n}^{2}+c k^{-1}\left\|\bar{U}_{h}^{n}-\tilde{U}_{h}^{n}\right\|_{n}^{2} \\
& +c k\left[\left(h^{r}+k^{\mu}+h k^{\mu-1 / 2}+h^{2} k^{\mu-1}\right)\left\|u^{0}\right\|_{\alpha}\right]^{2}, \\
& 0 \leq n \leq n^{*}-1 .
\end{aligned}
$$

Proposition 4.4. Let (1.22) be satisfied. Then there are constants $c_{0}>0$ and $\tilde{c}$ such that (4.13) holds. In fact, $\tilde{c}<0$ if (1.20) holds and $c(1)$ of (1.8) is small enough. 
Proof. See [14].

As with Proposition 3.2, $\tilde{c}<0$ is guaranteed for (4.13) by the following

PROPOSITION 4.5. Let (1.20) and (1.21) be satisfied. Then there are constants $c_{0}>0$ and $\tilde{c}<0$ such that (4.13) holds.

Proof. See [14].

Now, the terms of (4.12) are estimated as follows.

PROPOSITION 4.6. The terms of (4.12) satisfy

$$
\begin{aligned}
\left\|\bar{U}_{h}^{n}-\tilde{V}_{0}^{n}\right\|_{n} \leq & \sum_{m=n-1-\mu_{n}}^{n-1}\left\{\left\|\bar{U}_{h}^{m}-\tilde{U}_{h}^{m}\right\|_{m}+\left\|s^{m+1}-\varsigma^{m}\right\|_{m}\right\} \\
& +c k \sum_{m=n-1-\mu_{n}}^{n-1}\left\|\zeta^{m}\right\|_{m} \\
& +c k\left(h^{r}+k^{\mu_{n}}+h k^{\mu-1 / 2}+h^{2} k^{\mu-1}\right)\left\|u^{0}\right\|_{\alpha}, \\
& \quad 1 \leq n \leq n^{*}-1 .
\end{aligned}
$$

Proof. The techniques required are similar to those used for Proposition 3.3. See [14].

Next, (4.14) is combined with (4.9) for the estimation of the term $k^{-1}\left\|\bar{U}_{h}^{n}-\tilde{U}_{h}^{n}\right\|_{n}^{2}$ in (4.13).

PrOPOSITION 4.7. With $\bar{U}_{h}^{n}$ defined by (1.34) and $\tilde{U}_{h}^{n}$ by (1.39), the following hold:

$$
k^{-1}\left\|\bar{U}_{h}^{n}-\tilde{U}_{h}^{n}\right\|_{n}^{2} \leq c k^{-1} \beta_{n}^{2} \sum_{m=n-1-\mu_{n}}^{n-1}\left\{\left\|\bar{U}_{h}^{m}-\tilde{U}_{h}^{m}\right\|_{m}^{2}+\left\|\varsigma^{m+1}-\varsigma^{m}\right\|_{m}^{2}\right\}
$$

$$
\begin{aligned}
& +c k \beta_{n}^{2} \sum_{m=n-1-\mu_{n}}^{n-1}\left\|s^{m}\right\|_{m}^{2} \\
& +c k\left[\left(h^{r}+k^{\mu}+h k^{\mu-1 / 2}+h^{2} k^{\mu-1}\right)\left\|u^{0}\right\|_{\alpha}\right]^{2}, \\
& 1 \leq n \leq n^{*}-1,
\end{aligned}
$$

$$
\left\|\bar{U}_{h}^{0}-\tilde{U}_{h}^{0}\right\|_{0} \leq c k^{\mu}\left\|u^{0}\right\|_{\alpha} .
$$

Proof. By (1.39), (4.9) and (3.4),

$$
\left\|\bar{U}_{h}^{n}-\tilde{U}_{h}^{n}\right\|_{n} \leq c \beta_{n}^{l_{n}}\left\|\bar{U}_{h}^{n}-\tilde{V}_{0}^{n}\right\|_{n}, \quad 0 \leq n \leq n^{*}-1 .
$$

From (4.10) and (1.37) it follows that

$$
\beta_{n}^{l_{n}} k^{\mu_{n}} \leq c k^{\mu}, \quad 0 \leq n \leq n^{*}-1 .
$$

Finally, (4.15) follows after combining the last two inequalities with (4.14). Also, by (3.2), (3.20), and (3.13),

$$
\left\|\bar{U}_{h}^{0}-\tilde{V}_{0}^{0}\right\|_{0} \leq c\left\|U_{h}^{0}\right\|_{0} \leq c\left\|s^{0}\right\|_{0}+c\left\|\omega^{0}\right\|_{0} \leq c\left\|u^{0}\right\|_{\alpha}
$$

and (4.16) follows after combining this with the two estimates above.

Now, (4.15) demands an estimation of the differences $\left\|\zeta^{n+1}-\zeta^{n}\right\| n$, and this is the content of the following proposition. 
PROPOSITION 4.8. If (1.22) and (1.23) are satisfied, then the following holds:

$$
\begin{aligned}
& c_{1}\left\|\zeta^{n+1}-\varsigma^{n}\right\|_{n}^{2}+\left\|\left[I-r_{h}^{n+1}\right]^{1 / 2} \varsigma^{n+1}\right\|_{n+1}^{2} \\
& \leq c_{2} k^{2}\left\|s^{n}\right\|_{n}^{2}+c\left\|_{h}^{n}-\tilde{U}_{h}^{n}\right\|_{n}^{2}+\left(1+c_{3} k\right)\left\|\left[I-r_{h}^{n}\right]^{1 / 2} \varsigma^{n}\right\|_{n}^{2} \\
&+c k^{2}\left[\left(h^{r}+k^{\mu}+h k^{\mu-1 / 2}+h^{2} k^{\mu-1}\right)\left\|u^{0}\right\|_{\alpha}\right]^{2}, \quad 0 \leq n \leq n^{*}-1 .
\end{aligned}
$$

Proof. See [14].

Finally, the convergence result (1.5) is established for (1.39) as follows.

THEOREM 4.5. Let the conditions of either Proposition 4.4 or 4.5, in addition to those of Lemma 3.1 and Proposition 4.8, be satisfied. Then $\left\{j_{n}\right\}_{n=0}^{n^{*}-1}$ can be chosen so that (4.9) and (4.10) hold and provided $\varepsilon_{0}>0$ is small enough, the approximations $\left\{U_{h}^{n}\right\}_{n=0}^{n^{*}}$ obtained by (1.31) and (1.39) satisfy

$$
\max _{0 \leq n \leq n^{*}}\left\|U_{h}^{n}-u^{n}\right\| \leq c^{*}\left(h^{r}+k^{\mu}+h k^{\mu-1 / 2}+h^{2} k^{\mu-1}\right)\left\|u^{0}\right\|_{\alpha} .
$$

Also, unless $\tilde{c}<0$ in (4.13), $c^{*}$ depends exponentially on $t^{*}$.

Proof. Add $c_{1}\left\|\bar{U}_{h}^{n}-\tilde{U}_{h}^{n}\right\|_{n}^{2}$ to both sides of (4.17) and multiply the resulting inequality by $\varepsilon k^{-1} t^{n+1}$. When this is added to (4.13), the result is

$$
\begin{aligned}
&\left\|\zeta^{n+1}\right\|_{n+1}^{2}+c_{1} \varepsilon k^{-1} t^{n+1}\left\{\left\|\zeta^{n+1}-\varsigma^{n}\right\|_{n}^{2}+\left\|\bar{U}_{h}^{n}-\tilde{U}_{h}^{n}\right\|_{n}^{2}\right\} \\
& \quad+\varepsilon k^{-1} t^{n+1}\left\|\left[I-r_{h}^{n+1}\right]^{1 / 2} \varsigma^{n+1}\right\|_{n+1}^{2} \\
& \leq\left(1+\tilde{c} k+c_{2} \varepsilon k t^{n+1}\right)\left\|\zeta^{n}\right\|_{n}^{2}+c k^{-1}\left\|\bar{U}_{h}^{n}-\tilde{U}_{h}^{n}\right\|_{n}^{2} \\
&+c k\left[\left(h^{r}+k^{\mu}+h k^{\mu-1 / 2}+h^{2} k^{\mu-1}\right)\left\|u^{0}\right\|_{\alpha}\right]^{2} \\
&+\left[\varepsilon k^{-1} t^{n+1}\left(1+c_{3} k\right)-c_{0}\right]\left\|\left[I-r_{h}^{n}\right]^{1 / 2} \zeta^{n}\right\|_{n}^{2}, \quad 1 \leq n \leq n^{*}-1 .
\end{aligned}
$$

Now, for the compression of this inequality and others below, let the following be defined:

$$
\begin{array}{ll}
Z^{n} \equiv\left\|\zeta^{n}\right\|_{n}^{2}, & D^{n+1} \equiv\left\|\zeta^{n+1}-\zeta^{n}\right\|_{n}^{2}+\left\|\bar{U}_{h}^{n}-\tilde{U}_{h}^{n}\right\|_{n}^{2}, \\
S^{n} \equiv\left\|\left[I-r_{h}^{n}\right]^{1 / 2} \varsigma^{n}\right\|_{n}^{2}, & E \equiv\left[\left(h^{r}+k^{\mu}+h k^{\mu-1 / 2}+h^{2} k^{\mu-1}\right)\left\|u^{0}\right\|_{\alpha}\right]^{2} .
\end{array}
$$

With this notation, the following results after estimating $c k^{-1}\left\|\bar{U}_{h}^{n}-\tilde{U}_{h}^{n}\right\|_{n}^{2}$ with (4.15):

$$
\begin{aligned}
Z^{l+1}+ & c_{1} k^{-1} \varepsilon t^{l+1} D^{l+1}+\varepsilon k^{-1} t^{l+1} S^{l+1} \\
\leq & \left(1+\tilde{c} k+c_{2} \varepsilon k t^{*}\right) Z^{l}+c_{4} \beta_{l}^{2} \sum_{m=l-1-\mu_{l}}^{l-1}\left[k^{-1} D^{m+1}+k Z^{m}\right] \\
& +c k E+\left[\varepsilon k^{-1} t^{l}+\varepsilon\left(1+c_{3} t^{*}\right)-c_{0}\right] S^{l}, \quad 1 \leq l \leq n^{*}-1 .
\end{aligned}
$$

Now, assume that $\varepsilon>0$ is chosen small enough that $\varepsilon\left(1+c_{3} t^{*}\right) \leq c_{0}$. In fact, if $\tilde{c}<0$, suppose that $1+\tilde{c} k+c_{2} \varepsilon k t^{*} \leq 1+\hat{c} k$ for some $\hat{c} \leq 0$. Otherwise, take $\hat{c}>0$ in the following. Then, after summing the last inequality over $1 \leq l \leq n \leq n^{*}-1$, one gets

$$
\begin{aligned}
& \left(Z^{n+1}-Z^{1}\right)+\varepsilon k^{-1}\left(t^{n+1} S^{n+1}-t^{1} S^{1}\right)+c_{1} \varepsilon k^{-1} \sum_{l=1}^{n} t^{l+1} D^{l+1} \\
& \leq \hat{c} k \sum_{l=1}^{n} Z^{l}+c_{4} \sum_{l=1}^{n} \beta_{l}^{2} \sum_{m=l-1-\mu_{l}}^{l-1}\left[k Z^{m}+k^{-1} D^{m+1}\right] \\
& \quad+c t^{*} E, \quad 1 \leq n \leq n^{*}-1 .
\end{aligned}
$$


By (4.10) and (3.20), for $1 \leq n \leq n^{*}-1$,

$$
\begin{aligned}
\hat{c} k \sum_{l=1}^{n} Z^{l}+c_{4} k \sum_{l=1}^{n} \beta_{l}^{2} \sum_{m=l-1-\mu_{l}}^{l-1} Z^{m} & \leq\left(\hat{c}+c_{4} \varepsilon_{0}(\mu+1) t^{*}\right) k \sum_{l=1}^{n} Z^{l}+c k Z^{0} \\
& \leq \bar{c} k \sum_{l=1}^{n} Z^{l}+c k E,
\end{aligned}
$$

where $\bar{c} \leq 0$ if $\hat{c}<0$ and $\varepsilon_{0}>0$ is small enough. Otherwise, take $\bar{c}>0$ in the following. Next, since $(l+1) /(m+1) \leq \mu+2$ if $0 \leq l-1-\mu_{l} \leq m \leq l-1$, it follows, using (4.10), that for $1 \leq n \leq n^{*}-1$

$$
\begin{aligned}
c_{4} k^{-1} \sum_{l=1}^{n} \beta_{l}^{2} \sum_{m=l-1-\mu_{l}}^{l-1} D^{m+1} & \leq c_{4} k^{-1} \varepsilon_{0} \sum_{l=1}^{n} \sum_{m=l-1-\mu_{l}}^{l-1} \frac{l+1}{m+1} t^{m+1} D^{m+1} \\
& \leq c_{5} \varepsilon_{0} k^{-1} \sum_{l=1}^{n} t^{l+1} D^{l+1}+c D^{1}
\end{aligned}
$$

Combining the last three inequalities, one obtains for $1 \leq n \leq n^{*}-1$

$$
\begin{aligned}
Z^{n+1} & +\varepsilon k^{-1} t^{n+1} S^{n+1}+\left(c_{1} \varepsilon-c_{5} \varepsilon_{0}\right) k^{-1} \sum_{l=1}^{n} t^{l+1} D^{l+1} \\
& \leq\left(Z^{1}+\varepsilon S^{1}+c D^{1}\right)+c t^{*} E+\bar{c} k \sum_{l=1}^{n} Z^{l}
\end{aligned}
$$

By (3.4), (4.17), (4.16), (1.19) and (3.20), $Z^{1}+S^{1}+D^{1} \leq c E$. Now, assume that $\varepsilon_{0}>0$ is chosen small enough so that

$$
\begin{aligned}
\left\|\zeta^{n+1}\right\|_{n+1}^{2} \leq & c t^{*}\left[\left(h^{r}+k^{\mu}+h k^{\mu-1 / 2}+h^{2} k^{\mu-1}\right)\left\|u^{0}\right\|_{\alpha}\right]^{2} \\
& +\bar{c} k \sum_{l=0}^{n}\left\|\zeta^{l}\right\|_{l}^{2}, \quad 0 \leq n \leq n^{*}-1 .
\end{aligned}
$$

If $\bar{c} \leq 0$, ignore the last sum, and (4.18) follows after (1.12). If $\bar{c}>0$, then (4.18) follows with the discrete Gronwall Lemma and (3.20), but with $c^{*}$ depending exponentially on $t^{*}$.

5. Examples. The principal aim of this section is to present some computational results showing the strength of methods analyzed in this work. However, it is appropriate to first indicate that the set of IRKM's which satisfy the many conditions imposed in foregoing proofs is by no means vacuous. For example, in [16], it is explained that there exist $q$-stage methods of order $q+1$ and satisfying (1.20), (1.21), (1.24), (1.25), and (1.38), provided $q=1,2,3$, or 5. Furthermore, [16] gives explicit constructions of families of such methods for $q=2$ and 3. On the other hand, it is shown in [16] that for every positive integer $q$ there exists a collocation type IRKM satisfying (1.20), (1.24), (1.25), and (1.38).

As mentioned in the Introduction and more carefully in [16], the preferred methods in a parallel environment are those for which the eigenvalues of $A$ are distinct. These have been referred to as multiply implicit (MIRK) methods. Further, they are called real if $\sigma(A) \subset \mathbf{R}$, and otherwise complex. While the latter case has not been studied here, it is discussed in [16]. By considering that discussion together 
with the results of Bramble and Sammon [3], it can be seen that complex MIRK's can be analyzed using quadratic preconditioning and hence inverse assumptions.

In contrast to MIRK's there are the well-known methods for which the eigenvalues of $A$ are identical and real, [12]. As seen in (4.7), these so-called singly implicit (SIRK) methods offer a computational advantage on serial machines since at each time step, they require the formation of only a single new matrix with the dimension of $S_{h}$. A selection from this set of methods was made for the example considered below.

The following problem is of the class defined in the Introduction:

$$
\begin{cases}\partial_{t} u=-L(t) u & \text { in }(-1,1) \times[0, .1] \\ u=0 & \text { on }\{-1,1\} \times[0, .1] \\ u(x, 0)=1-x^{2} & \text { in }(-1,1)\end{cases}
$$

where

$$
\begin{aligned}
L(t) u & \equiv-\partial_{x}\left(\ell_{1}(x, t) \partial_{x} u\right)+\ell_{0}(x, t) u \\
\ell_{1}(x, t) & \equiv \frac{\frac{1}{18} \log (2)\left(3-x^{2}\right)}{\left(2+x^{2}\right)+t\left(1-x^{2}\right)}\left(2+x^{2}\right)^{t+1} \\
\ell_{0}(x, t) & \equiv \log \left(2+x^{2}\right)-\frac{1}{3} \log (2)\left(2+x^{2}\right)^{t}
\end{aligned}
$$

The solution is given by

$$
u(x, t)=\frac{1-x^{2}}{\left(2+x^{2}\right)^{t}} .
$$

For the spatial discretization, the Ordinary Galerkin Method was used and $S_{h}$ was

\begin{tabular}{|c|c|c|c|}
\hline$k, h$ & CPU Time (sec) & $L_{2}$ Error $\left(\times 10^{9}\right)$ & Order \\
\hline $1 / 50$ & 22 & 1.19 & \\
\hline $1 / 60$ & 30 & .525 & 4.49 \\
\hline $1 / 70$ & 38 & .266 & 4.42 \\
\hline $1 / 80$ & 48 & .148 & 4.37 \\
\hline $1 / 90$ & 59 & .0889 & 4.33 \\
\hline $1 / 100$ & 72 & .0565 & 4.30 \\
\hline
\end{tabular}
constructed of smooth cubic splines defined on a uniform mesh. For the temporal discretization, the well-known three-stage diagonally implicit (DIRK) method was used, as it satisfies (1.20), (1.21), (1.24), and (1.25). ([8], [15])

TABLE 1. Modified method

Now let (1.39) be identified as the modified method, and an analogue based on (1.33) as the classical method. In addition, let a hybrid method be given by (1.39), but with $D^{l}$ replaced by $T^{l}$ in (1.28). These three methods were tested 
on a SUN 3/180 with FPA. Defining $E(h, k) \equiv\left\|U_{h}^{n^{*}}-u^{n^{*}}\right\|$, the $L_{2}$ errors $E(k) \equiv$ $E(k, k)$ are reported in Tables 1-3, together with estimates of the convergence order obtained according to the formula $\log \left(E\left(k_{2}\right) / E\left(k_{1}\right)\right) / \log \left(k_{2} / k_{1}\right)$.

With regard to time consumption, recall that the computational burden for the classical method is in forming $q$ new stiffness matrices at each time step. On the other hand, with the constants $\left\{\delta_{m}^{n}\right\}_{0 \leq m \leq n-1}^{0 \leq n \leq n^{*}-1}$ given by (1.30), the burden for the modified method is in forming the terms $\phi_{i}$ of (4.5), for the right side of (4.7). Also, the initial step is relatively expensive, but the effect of this diminishes as the number of time steps increases. Note that among the three methods tested, numbers for the modified method were obtained with greater speed and accuracy, as well as with fourth-order convergence. On the other hand, the others suffer from suboptimal convergence as explained in the Introduction. However, no rigorous explanation can be offered for the identical accuracy obtained by the classical and hybrid methods. Further, this author is unaware of any proof of the better than second-order convergence seen in Tables 2 and 3 . In this connection, note that the

TABLE 2. Classical method

\begin{tabular}{|c|c|c|c|}
\hline$k, h$ & CPU Time (sec) & $L_{2}$ Error $\left(\times 10^{9}\right)$ & Order \\
\hline $1 / 50$ & 22 & 28.5 & \\
\hline $1 / 60$ & 31 & 16.0 & 3.16 \\
\hline $1 / 70$ & 41 & 9.80 & 3.19 \\
\hline $1 / 80$ & 52 & 6.36 & 3.23 \\
\hline $1 / 90$ & 65 & 4.35 & 3.24 \\
\hline $1 / 100$ & 77 & 3.09 & 3.24 \\
\hline
\end{tabular}

TABLE 3. Hybrid method

\begin{tabular}{|c|c|c|c|}
\hline$k, h$ & CPU Time (sec) & $L_{2} \operatorname{Error}\left(\times 10^{9}\right)$ & Order \\
\hline $1 / 50$ & 23 & 28.3 & \\
\hline $1 / 60$ & 30 & 15.8 & 3.21 \\
\hline $1 / 70$ & 38 & 9.61 & 3.21 \\
\hline $1 / 80$ & 49 & 6.25 & 3.22 \\
\hline $1 / 90$ & 59 & 4.28 & 3.22 \\
\hline $1 / 100$ & 71 & 3.04 & 3.23 \\
\hline
\end{tabular}


above solution has no time derivatives which are even in the domain of $L(t)^{2}$, a condition considered necessary to escape order reduction in a general way. Nevertheless, only second-order convergence is demonstrated, for example in Experiment 7.5.1 of Dekker and Verwer [8], where a stiff ordinary differential equation is considered. Further, the modified method has been applied to this problem to give not only fourth-order convergence, but accuracy exceeding that reported for any method discussed in the Experiment.

Department of Mathematics

Vanderbilt University

Nashville, Tennessee 37235

E-mail: keeling@math1.cas.vanderbilt.edu

1. R. A. Adams, Sobolev Spaces, Academic Press, New York, 1975.

2. L. A. BALES, "Semidiscrete and single step fully discrete approximations for second order hyperbolic equations with time-dependent coefficients," Math. Comp., v. 43, 1984, pp. 383-414.

3. J. H. BRAMBLE \& P. H. SAMMON, "Efficient higher order single step methods for parabolic problems: Part I," Math. Comp., v. 35, 1980, pp. 655-677.

4. J. H. Bramble, A. H. Schatz, V. Thomée \& L. B. Wahlbin, "Some convergence estimates for semidiscrete Galerkin type approximations for parabolic equations," SIAM J. Numer. Anal., v. 14, 1977, pp. 218-241.

5. J. C. ButChER, "Implicit Runge-Kutta processes," Math. Comp., v. 18, 1964, pp. 50-64.

6. P. G. Ciarlet, The Finite Element Method for Elliptic Problems, North-Holland, Amsterdam, 1978.

7. M. Crouzeix, Sur l'Approximation des Équations Différentielles Opérationnelles Linéaires par des Méthodes de Runge-Kutta, Thèse, Université de Paris VI, 1975.

8. K. DEKKer \& J. G. VerWER, Stability of Runge-Kutta Methods for Stiff Nonlinear Differential Equations, North-Holland, Amsterdam, 1984.

9. V. A. Dougalis \& O. A. KaRAKashian, "On some high-order accurate fully discrete Galerkin methods for the Korteweg-De Vries equation," Math. Comp., v. 45, 1985, pp. 329-345.

10. J. Douglas, JR., T. DUPONT \& R. EWING, "Incomplete iterations for time-stepping a Galerkin method for a quasilinear parabolic problem," SIAM J. Numer. Anal., v. 16, 1979, pp. 503-522.

11. L. A. Hageman \& D. M. Young, Applied Iterative Methods, Academic Press, New York, 1981.

12. E. HAIRER \& G. WANNER, "Algebraically stable and implementable Runge-Kutta methods of high order," SIAM J. Numer. Anal., v. 18, 1981, pp. 1098-1108.

13. O. A. KarAKaShian, "On Runge-Kutta methods for parabolic problems with timedependent coefficients," Math. Comp., v. 47, 1986, pp. 77-106.

14. S. L. Keeling, Galerkin/Runge-Kutta Discretizations for Parabolic Equations with Time Dependent Coefficients, ICASE Report No. 87-61, NASA Langley Research Center, Hampton, VA, 1987.

15. S. L. Keeling, Galerkin/Runge-Kutta Discretizations for Parabolic Partial Differential Equations, Ph.D. Dissertation, University of Tennessee, 1986.

16. S. L. KeEling, On Implicit Runge-Kutta Methods for Parallel Computations, ICASE Report No. 87-58, NASA Langley Research Center, Hampton, VA, 1987.

17. P. H. SAMmon, Approximations for Parabolic Equations with Time-Dependent Coefficients, Ph.D. Thesis, Cornell University, 1978.

18. P. H. SAMMON, "Convergence estimates for semidiscrete parabolic equation approximations," SIAM J. Numer. Anal., v. 19, 1982, pp. 68-92. 